\title{
A novel antibody against cancer stem cell biomarker, DCLK1-S, is potentially useful for assessing colon cancer risk after screening colonoscopy
}

\author{
Shubhashish Sarkar', Vsevolod L Popov², Malaney R O'Connell' ${ }^{1}$, Heather L Stevenson², Brian S Lee ${ }^{3}$, Robert A Obeid ${ }^{3}$, \\ Gurinder K Luthra ${ }^{4}$ and Pomila Singh ${ }^{1}$
}

DCLK1 expression is critically required for maintaining growth of human colon cancer cells (hCCCs). Human colorectal tumors (CRCs) and hCCCs express a novel short isoform of DCLK1 (DCLK1-S; isoform 2) from $\beta$-promoter of hDCLK1 gene, while normal colons express long isoform (DCLK1-L; isoform 1) from $5^{\prime}(a)$-promoter, suggesting that DCLK1-S, and not DCLK1-L, marks cancer stem cells (CSCs). Even though DCLK1-S differs from DCLK1-L by only six amino acids, we succeeded in generating a monospecific DCLK1-S-Antibody (PS41014), which does not cross-react with DCLK1-L, and specifically detects CSCs. Subcellular localization of S/L-isoforms was examined by immune-electron-microscopy (IEM). Surprisingly, besides plasma membrane and cytosolic fractions, S/L also localized to nuclear/mitochondrial fractions, with pronounced localization of S-isoform in the nuclei and mitochondria. Sporadic CRCs develop from adenomas. Screening colonoscopy is used for detection/resection of growths, and morphological/pathological criteria are used for risk assessment and recommendations for follow-up colonoscopy. But, these features are not precise and majority of the patients will never develop cancer. We hypothesized that antibody-based assay(s), which identify CSCs, will significantly improve prognostic value of morphological/pathological criteria. We conducted a pilot retrospective study with PS41014$\mathrm{Ab}$, by staining archived adenoma specimens from patients who developed (high-risk), or did not develop (low-risk) adenocarcinomas within 10-15 years. PS41014-Ab stained adenomas from initial and follow-up colonoscopies of high-risk patients, at significantly higher levels (three to fivefold) than adenomas from low-risk patients, suggesting that PS41014Ab could be used as an additional tool for assessing CRC risk. CRC patients, with high DCLK1-S-expressing tumors (by qRT-PCR), were reported to have worse overall survival than low expressers. We now report that DCLK1-S-specific Ab may help to identify high-risk patients at the time of index/screening colonoscopy.

Laboratory Investigation (2017) 97, 1245-1261; doi:10.1038/labinvest.2017.40; published online 17 April 2017

Colorectal cancers (CRCs) are one of the leading causes of death due to cancers. ${ }^{1}$ More than 100000 people die every year due to CRC in the United States alone. ${ }^{1}$ All CRCs develop from premalignant adenomatous (Ads) polyps in the colon. $^{2}$ Adults, aged $\geq 50$ years, with no genetic predisposition, develop sporadic hyperplastic (Hps) and adenomatous growths; by age $60,30-40 \%$ of patients have colonic Ads. However, only $\sim 5 \%$ of these patients will develop malignant growths in their lifetime. ${ }^{2-4}$ As a preventive measure, screening colonoscopy is recommended for all adults over the age of 50. ${ }^{5}$ Follow-up colonoscopies are recommended at intervals of every 2-10 years, based on risk assessment.
Currently, patients positive for colonic polyps are assigned either high or low risk, based on number $(\geq 3 /<3)$ and size $(\geq 1 \mathrm{~cm} /<1 \mathrm{~cm})$ of polyps, and presence/absence of highgrade dysplasia. Follow-up intervals of $\sim 5-10$ years are recommended for patients positive for only $\mathrm{Hps}$ with no evidence of dysplasia. ${ }^{4-6}$ However, $<10 \%$ high-risk patients will eventually develop CRCs, while $\sim 10-20 \%$ of low-risk patients develop malignant adenocarcinomas (AdCAs), including advanced Ads (A-Ads) of clinical concern. ${ }^{6-8}$ Thus, there is a critical need for developing molecular biomarkers, besides currently used morphological/pathological qualifiers, to better identify patients at risk of developing AdCAs. An

\footnotetext{
'Departments of Neuroscience and Cell Biology, University of Texas Medical Branch, Galveston, TX, USA: ${ }^{2}$ Department of Pathology, University of Texas Medical Branch Galveston, TX, USA; ${ }^{3}$ University of Texas Medical Branch, Galveston, TX, USA and ${ }^{4}$ Gastrointestinal Division, Department of Internal Medicine, University of Texas Medical Branch, Galveston, TX, USA

Correspondence: Professor P Singh, PhD, Departments of Neuroscience and Cell Biology, Biochemistry and Molecular Biology and Internal Medicine, University of Texas Medical Branch, Galveston, TX, USA.

E-mail: posingh@utmb.edu

Received 17 November 2016; revised 1 March 2017; accepted 2 March 2017
} 
accurate identification can potentially reduce high rates of false positive/negative recommendations, and reduce overall burden of repeated colonoscopies for majority of the patients who will never develop CRCs. ${ }^{9,10}$ As transformation of normal stem cells (NSCs) to cancer stem cells (CSCs) can increase the risk of forming malignant growths (discussed in refs 11, 12), we hypothesized that presence of CSCs in resected polyps may predict poor outcomes for patients, irrespective of morphological or pathological features of the polyps.

Several CSC markers have been identified, including CD44, CD133, Lgr5, and DCLK1, which are reported to have a functional role in maintaining growth and/or metastatic spread of cancer cells (described in ref. 12). DCLK1 was recently identified as a colon cancer-specific marker, and has a critical role in colon/pancreatic carcinogenesis in mice. ${ }^{13,14}$ DCLK1 is also critically required for maintaining proliferative potential of human colon cancer cells (hCCCs). ${ }^{15}$ DCLK1 expression is upregulated in response to growth factors, resulting in hyperproliferation of colonic crypts in mice. ${ }^{11} \mathrm{We}$ recently reported that subsets of DCLK1+CSCs are resistant to inhibitory effects of chemoprevention/chemotherapeutic agents; downregulation of DCLK1 was required for eliminating CSCs in response to chemopreventive/chemotherapeutic agents. ${ }^{15}$ However, isogenic clones of human embryonic epithelial cells, either poorly tumorigenic or highly metastatic, expressed identical set of stem cell markers, including DCLK1. ${ }^{16}$ Thus, it has remained a challenge to specifically target CSCs, while sparing normal cells, to avoid toxicity. Our recent findings, ${ }^{12}$ as described below, will likely allow identification of CSCs in colonic tumors, at the time of screening colonoscopy itself, and help to improve the accuracy of follow-up colonoscopy recommendations.

The 5'-promoter of human (h)DCLK1 gene is epigenetically silenced in hCRCs. ${ }^{12,17-20}$ Hypermethylation of $5^{\prime}$ promoter was reported to be an early event during adenomacarcinoma sequence of colon-carcinogenesis in humans. ${ }^{12}$ As a result, the long transcripts/isoforms of hDCLK1 are not expressed in hCRCs. ${ }^{12}$ Even though the $5^{\prime}$-promoter of $\mathrm{h} D C L K 1$ gene is epigenetically silenced, ${ }^{12}$ high levels of DCLK1 protein are present in hCRCs (discussed in ref. 12). To address the discrepancy between the reported presence of DCLK1 protein in hCRCs, but epigenetic silencing of $5^{\prime}$-promoter in hCRCs, possible use of an alternate promoter for expressing alternate isoforms of DCLK1 was investigated. Human colon cancer cells (hCCCs) and CRCs were discovered to express short transcripts of DCLK1 (DCLK1$\mathrm{S}$; isoform 2 in the NCBI database) from an alternate $\beta$-promoter in IntronV of the gene, while normal colons mainly expressed the long isoform of DCLK1 (DCLK1-L; isoform 1 in the NCBI database) from $5^{\prime} \alpha$-promoter, ${ }^{12}$ as recently reviewed. ${ }^{20}$ Thus our findings in the past few years, suggested that DCLK1-S may represent a CSC-specific marker in humans, while DCLK1-L mainly marks normal human cells. Pathophysiological relevance of DCLK1-S expression by
hCRCs was examined in a cohort of 92 CRC patients; high expressers had significantly worse overall survival and diseasefree interval compared with low expressers. ${ }^{12}$ Importantly, DCLK1-S expression was found to represent an independent diagnostic/prognostic marker for CRC patients. ${ }^{12}$ These findings led us to develop a monospecific antibody $(\mathrm{Ab})$ against the unique CSC-specific marker, DCLK1-S.

Several antibodies have been developed against the C-terminal end of DCLK1 proteins, which is common to both the short and long isoforms (described in ref. 12). Investigators in the field have used commercially available antibodies against the common C-terminal end of DCLK1 to identify the presence of DCLK1 in normal and/or cancer cells. ${ }^{11-16,21-29}$ Antibodies against DCLK1-L, generated against epitopes within the double-cortin (DCX) domains of DCLK1-L, at the N-terminal end of the protein, have also become available, and specifically identify the L-isoform, because short isoforms, including isoform 2, lack DCX domains (described in ref. 12). Even though isoforms 1 and 2 have been described in neuronal cells, possible differential effects of the isoforms, remains unknown. Specific antibodies against the short isoform are not available. As human epithelial cancers (colon/pancreatic) mainly express the Sisoform, ${ }^{12,30}$ representing a CSC-specific biomarker, we generated a monospecific antibody against the unique amino acids at the $\mathrm{N}$-terminal end of the short isoform. In previous years, the short isoform present in the neuronal cells was believed to represent a proteolytic fragment of the L-isoform due to enzymatic processing by calpain enzyme. ${ }^{31}$ Although it remains possible that $\mathrm{L}$-isoform derived fragments are also present in epithelial cells, our studies strongly suggest that short fragments of DCLK1 in human colon/pancreatic cancer cells, are the product of a unique S-transcript, transcriptionally derived from the $\beta$-promoter of $\mathrm{h} D C L K 1$ gene. ${ }^{12}$ The S-transcript is $>98 \%$ homologous with the $3^{\prime}$-end of the L-transcript, ${ }^{12}$ but has unique nt sequences at the $5^{\prime}$-end, resulting in the presence of six unique amino acids at the $\mathrm{N}$-terminal end of DCLK1-S protein. We took advantage of the unique moieties, and generated a monospecific antibody against the S-isoform of DCLK1, as reported in here. The specificity/sensitivity of the antibody was confirmed in the current studies. As the S-isoform lacks DCX domains, we hypothesized that the intracellular localization of the two isoforms may be different. Electron microscopy (EM) was used to identify possible differential localization of the isoforms in isogenic clones of colon cancer cells, expressing either the L or S-isoforms. Our studies demonstrate that the isoforms are not only present at the plasma membranes and in the cytosol of cancer cells, but are also present in the nuclei and mitochondria of the cells.

To determine whether DCLK1-S can potentially serve as a biomarker at the time of screening colonoscopy, as proof of principle, we conducted a pilot retrospective study with antiDCLK1-S antibody $(\mathrm{Ab})$, generated by our laboratory. Our findings suggest that DCLK1-S can be used as a biomarker, at 
the time of index/screening colonoscopy, for identifying high- $v$ s low-risk patients, more accurately, than the currently used morphological/pathological criteria. The discovery of DCLK1-S as a specific marker of CSCs in human colonic tumors $^{12}$ provides an opportunity for identifying the small subset of high-risk patients who will likely develop malignant growths within a shorter time span, and who may benefit from aggressive management to prevent onset of the CRC disease.

\section{MATERIALS AND METHODS Reagents Used}

Antibodies (Abs) used in these studies included: anti-DCLK1 (generated against the common C-terminal end of the molecule, which is common to all the isoforms; Abcam, Ab31704); anti-DCLK1-L antibody (generated against an epitope within the double-cortin domains and specific for the long isoform; Abcam, Ab106635); anti- $\beta$-actin (total; Sigma, St. Louis, MO, USA); rabbit polyclonal antibody conjugated to Horseradish peroxidase (GE Healthcare, UK); goat antirabbit immunogold (15 nM) antibody (Electron Microscopy Sciences, Hatfield, PA, USA). Reagents used in these studies included $\mathrm{ABC}$ staining kit for immunohistochemistry (Vector Laboratories, Burlington, CA, USA) and TSA Plus Biotin Kit (PerkinElmer, Waltham, MA, USA). Transfection reagent, FuGENE6 was bought from Roche (Branford, CT, USA). All reagent-grade chemicals were purchased from Sigma.

\section{Generation of Monospecific, Polyclonal Antibody, Against DCLK1-S Isoform}

The DCLK1-S isoform shares $>98 \%$ amino-acid homology with the C-terminal domains of the DCLK1-L isoform. However, DCLK1-S differs from DCLK1-L at the N-terminal end by six unique amino acids (Supplementary Figure 1). These unique amino acids were used as an antigen. To increase immunogenicity of the synthetic six amino-acid peptide, eight copies of the peptide were displayed on a lysine branched scaffold using multiple antigen peptide octavalent (MAP-8). The peptide and MAP-8 complex was purified and dissolved in $50 \%$ DMSO followed by mixing with complete Freund's adjuvant for immunizing the rabbits. After five booster immunizations, antisera was collected after 16 weeks of initiating immunizations. The antisera were purified using immunoaffinity column generated by coupling the antigen to sepharose beads to yield monospecific polyclonal antibody to DCLK1-S. The sensitivity and specificity of the antibody (PS41014), thus generated, was examined as described under the 'Results' section.

\section{Cell Culture}

The HCT116 and HEK293 cell lines were obtained from ATCC (Manassas, VA, USA), and have been maintained in the laboratory for several years. All other colon cancer cell lines were purchased from ATCC within the past 2 years and confirmed by ATCC. All cell lines were monitored regularly for absence of mycoplasma. The HCT116 and HEK293 cell lines were confirmed to represent human epithelial cell lines with the help of Biosynthesis Company (Lewisville, TX, USA). HEKC and HEKmGAS stable clones were generated to overexpress either control (HEKC) or triple mutant human gastrin gene to overexpress full-length progastrin peptide (HEKmGAS), as previously described. ${ }^{16}$ All cell lines were cultured in DMEMF12 medium (Invitrogen, Grand Island, NY, USA), supplemented with $10 \%$ FCS containing $1 \%$ penicillin/streptomycin in a humid atmosphere at $37^{\circ} \mathrm{C}$ with $5 \% \mathrm{CO}_{2}$. The stable clones of HEK293 (HEKC; HEKmGAS) and COLO205 cells (described below), were cultured in the same medium, supplemented with $100 \mu \mathrm{g} / \mathrm{ml}$ Geneticin (Invitrogen) under similar conditions.

\section{Generation of COLO205 Clones, Stably Overexpressing Full-Length GFP-DCLK1-L/S}

Eukaryotic expression plasmids expressing $\mathrm{N}$-terminal GFP-tagged full-length coding sequences of either DCLK1-L or DCLK1-S were purchased (GeneCopoeia, Rockville, MD, USA). COLO205 clones stably expressing full-length DCLK1L (205-L) or DCLK1-S (205-S) were generated as previously described. ${ }^{16}$ Vector Transfected clones (205-C) expressing only GFP served as controls. GFP and DCLK1 expression by the clones was confirmed by western blot analysis.

\section{Procurement of Human Patient Samples}

Human tissues samples were collected as per our approved IRB protocols (UTMB-IRB \#03-237, UTMB-IRB \#91-310), as previously described. ${ }^{12}$ Normal colonic mucosa samples were collected from consented patients at the time of endoscopy, only if patients were free of adenomas (Ads) or adenocarcinomas (AdCas), but positive for small $\mathrm{Hp}$ growths. Tumor samples were obtained as discarded specimens from primary adenocarcinomas or metastatic colonic growths with the help of Tissue Core Facility at the Cancer Center in the University of Alabama. All the samples were snap frozen in liquid nitrogen and stored at $-80^{\circ} \mathrm{C}$ till further processing. Colonic adenoma (Ad) samples, fixed in formalin and embedded in paraffin (FFPE blocks) were also obtained as archived specimens from the Department of Pathology at UTMB. The histopathologic features (eg, Hps, Ads, AdCAs) in each of the specimen blocks was confirmed by evaluation of $\mathrm{H} \& \mathrm{E}$ sections by Dr Stevenson, a board-certified pathologist.

\section{Western Immunoblot Analysis}

Cells growing as monolayer cultures at $60-70 \%$ confluency, were collected and processed for preparing cellular lysates as previously described. ${ }^{11,12,16}$ Frozen tissue patient samples, obtained as described above, were homogenized and processed for preparation of tissue lysates in RIPA buffer as described previously. ${ }^{12}$ Samples containing $30-50 \mu \mathrm{g}$ of proteins were subjected to electrophoresis and transferred to PVDF membranes. The blots were cut into horizontal strips containing target or loading-control proteins ( $\beta$-actin), and 
processed for western immunoblot (WB) analysis. Antigen antibody complexes were detected with a chemiluminescencereagent kit (Thermoscientific, IL, USA or GE Healthcare). Membrane strips containing either target or loading-control proteins were simultaneously exposed for equal time to autoradiographic films. Western blots presented were cropped to exclude bands beyond the range of the molecular markers, at the running and loading ends. Processing of films was applied equally across the entire image. Touch-up tools were not used to manipulate data.

\section{Immunostaining}

For IHC staining of patient tissue samples, slides containing tissue sections were deparaffinized and hydrated using xylene and ethanol. The slides were unmasked for antigen by boiling in sodium citrate buffer, $\mathrm{pH}$ 6.0. The sections were washed and $5 \%$ goat serum was used to block nonspecific binding. The commercially purchased antibodies, that were either specific for DCLK1-L isoform or common to all the isoforms, were used at the dilution suggested by the manufacturer. PS41014-Ab, generated by our laboratory, was used at variable dilutions (1:50-1:1000) and 1:200 dilution was determined to be optimal for IHC staining (data not shown). Thus the PS41014-Ab was used at 1:200 dilution for the IHC studies, presented in here, and the slides were incubated with the primary $\mathrm{Ab}$ at $4^{\circ} \mathrm{C}$ overnight. Incubated sections were washed and incubated at room temperature (RT) for $2 \mathrm{~h}$ with HRP-conjugated anti-rabbit-IgG at 1:200 dilution. The sections were further incubated for $30 \mathrm{~min}$ at RT, using biotinylated secondary anti-rabbit or anti-mouse antibodies (1:200), followed by washing with PBS and incubated with $\mathrm{DAB}$ (ABC kit, Vector Laboratories). The sections were stained with hematoxylin $(\mathrm{H})$ and then mounted. Images from the tissue sections were captured by a Nikon microscope (TS100) equipped with camera at $\times 10, \times 20$, and $\times 40$ magnifications, after mounting the sections with coverslips, as previously described. ${ }^{32,33}$ The $\%$ area or $\%$ epithelial cells, specifically stained with the antibodies, was determined as described in the legends of the figures.

\section{Immuno-Electron Microscopy}

The methods used for Immuno-Electron (EM) microscopy are similar to methods we have previously used in our publications. $^{34,35}$ Briefly, the cells growing as monolayer cultures at $60-70 \%$ confluency were collected and fixed in $2.5 \%$ formaldehyde and $0.1 \%$ glutaraldehyde in $0.05 \mathrm{M}$ cacodylate buffer pH 7.2 containing $0.01 \%$ trinitrophenol and $0.03 \% \mathrm{CaCl}_{2}$ for $2 \mathrm{~h}$ at RT. The cells were washed in $0.1 \mathrm{M}$ cacodylate buffer cells and then scraped and pelleted. The pelleted cells were stained en bloc with $2 \%$ aqueous uranyl acetate, dehydrated in 50 and $75 \%$ ethanol and embedded in LR White resin medium grade (Electron Microscopy Sciences, Hatfield, PA, USA). Ultrathin sections were cut on Leica EM UC7 ultramicrotome (Leica Biosystems, Buffalo Grover, IL, USA) and collected onto Formvar- carbon-coated nickel grids (Electron Microscopy Sciences). The grids were incubated in blocking buffer $(0.1 \%$ BSA and $0.01 \mathrm{M}$ glycine in $0.05 \mathrm{M}$ tris-buffered saline (TBS)), followed by primary antibody (anti-GFP or PS41014-Ab) in 1\% BSA in $0.05 \mathrm{M}$ TBS (diluting buffer) for $1 \mathrm{~h}$ at RT and then overnight at $4{ }^{\circ} \mathrm{C}$. The grids were washed and incubated with secondary antibody (goat anti-rabbit IgG) conjugated to $15 \mathrm{~nm}$ colloidal gold particles at a dilution of 1:20, for $1 \mathrm{~h}$ at room temperature. The grids were washed in TBST buffer and water and fixed in $2 \%$ aqueous glutaraldehyde. The grids were then washed and stained with uranyl acetate and lead citrate and examined in a Philips (FEI) 201 or Philips (FEI) CM-100 transmission electron microscope (Hillsboro, OR, USA) at $60 \mathrm{kV}$.

\section{Design of the Pilot Retrospective Study}

To assess the predictive value of DCLK1-S staining in resected patient polyps at the time of baseline colonoscopy, we designed a pilot retrospective study. Archived FFPE samples were obtained from the Department of Pathology at UTMB. We have an approved IRB Protocol (91-310) for discarded specimens of colonic growths (Hps, Ads, and AdCAs). Two databases were available to us for selecting FFPE blocks that can be potentially assigned to either the low-risk group or the high-risk group. The two databases were arbitrarily termed the polyp database and the CRC database.

The polyp database lists the colonoscopy reports of all patients who received colonoscopies since 1992. Using the polyp database from 2000 to 2001 alone, we identified $>400$ patients who returned for follow-up colonoscopies for $\geq 10$ years. A digitized database of polyp samples, collected since 1992, was available to us. The UH numbers of the identified patients were used to print PDI (previous diagnostic inquiry) reports, which contain pathology reports from all colonoscopies conducted at the UTMB. Majority of the patients remained free of any significant growths, but at least $20-30 \%$ were positive for adenomas at index and follow-up colonoscopies, but remained free of A-Ads/CRCs during this period. We selected six patients from this group, as representative of low-risk group, as none of them developed A-Ads or AdCAs during the follow-up period. The CRC database was used for identifying patient samples for the high-risk arm of the retrospective study; CRC database lists all patients who had surgical removal of CRCs at UTMB. We screened the 2014-2015 database of CRC patients, and identified several patients who had prior history of colonoscopies at UTMB, dating back to $>8-12$ years. Many of these patients were positive for only benign growths at the time of index/followup colonoscopies, and were selected as examples of high-risk patients. Patient FFPE blocks were obtained, irrespective of ethnicity, age, and gender. The ethnic mix of patients reflects the population of Galveston County in TX, USA. The number, size (when available), and pathology of all polyps that were collected at each colonoscopy/surgery for the low- 
and high-risk arms of the study, was tabulated and is presented in Supplementary Tables.

On average, slides with consecutive tissue sections were prepared from approximately three to seven FFPE blocks from each patient (to include at least two Ad specimens, eg, from initial and follow-up colonoscopies). At least one slide/ polyp was stained with H\&E to confirm morphology/ pathology as described in the reports. The remaining slides were processed for IHC by our published procedures. ${ }^{32,33}$ The sections were stained with DCLK1-S-Ab (PS41014) in duplicate. The imageJ method, with conversion of positive staining to red scale was used for quantifying the relative staining of the specimens, in terms of $\%$ area stained. The $\%$ epithelial cells stained was also analyzed. The highest stained polyp from an index and follow-up colonoscopy was used for purposes of comparison.

\section{Statistical Analysis}

The data are presented as mean \pm s.e.m. of values obtained from indicated number of patient samples or experiments. To test for significant differences between means, nonparametric Mann-Whitney test using STAT view 4.1 (Abacus Concepts, Berkley, CA, USA) was used. The $P$-values $<0.05$ were considered significant.

\section{RESULTS \\ Specificity and Sensitivity of Anti-DCLK1-S Antibody (PS41014) for Detecting Short Isoform (Isoform 2) of hDCLK1}

As described under the 'Materials and Methods' section, we generated a monospecific polyclonal antibody (Ab; PS41014) against a multimer of the unique $\mathrm{N}$-terminal peptide sequence of DCLK1-S. Isogenic clones of HEK293 cells, which either expressed only the L-isoform (HEKC, HEK293) or both the L/S-isoforms (HEKmGAS) ${ }^{12}$ were used. A representative colon cancer cell line, HCT116, which only expresses the S-isoform, due to epigenetic silencing of the $5^{\prime}$-promoter, ${ }^{12}$ was also used. To validate the specificity of the PS41014-Ab, commercially available antibodies that either detect all isoforms of DCLK1 (Ab31704), or detect only the L-isoform (Ab106635) were used. The data with all three antibodies is shown in Figures $1 \mathrm{a}-\mathrm{c}$. As expected, the long specific $\mathrm{Ab}$ detected the presence of L-isoform $(\sim 80 \mathrm{kDa})$ by WB analysis of the lysate samples prepared from HEKC and HEK293 cells (Figure 1a). As human colon cancer cell lines (hCCCs), including HCT116, do not express the L-isoform, the long specific Ab did not detect the S-isoform in HCT116 cells, further validating our previous findings demonstrating presence of only the S-isoform in the majority of hCCCs. ${ }^{12}$ The L-specific Ab, however, did detect a smaller band in HEKmGAS cells, which may represent a fragment of the $\mathrm{C}$-terminal end of L-isoform, as the long specific Ab was generated against an epitope within the C-terminal doublecortin domains of L-isoform. The same cellular samples were next probed with the common $\mathrm{Ab}$, which detects both the
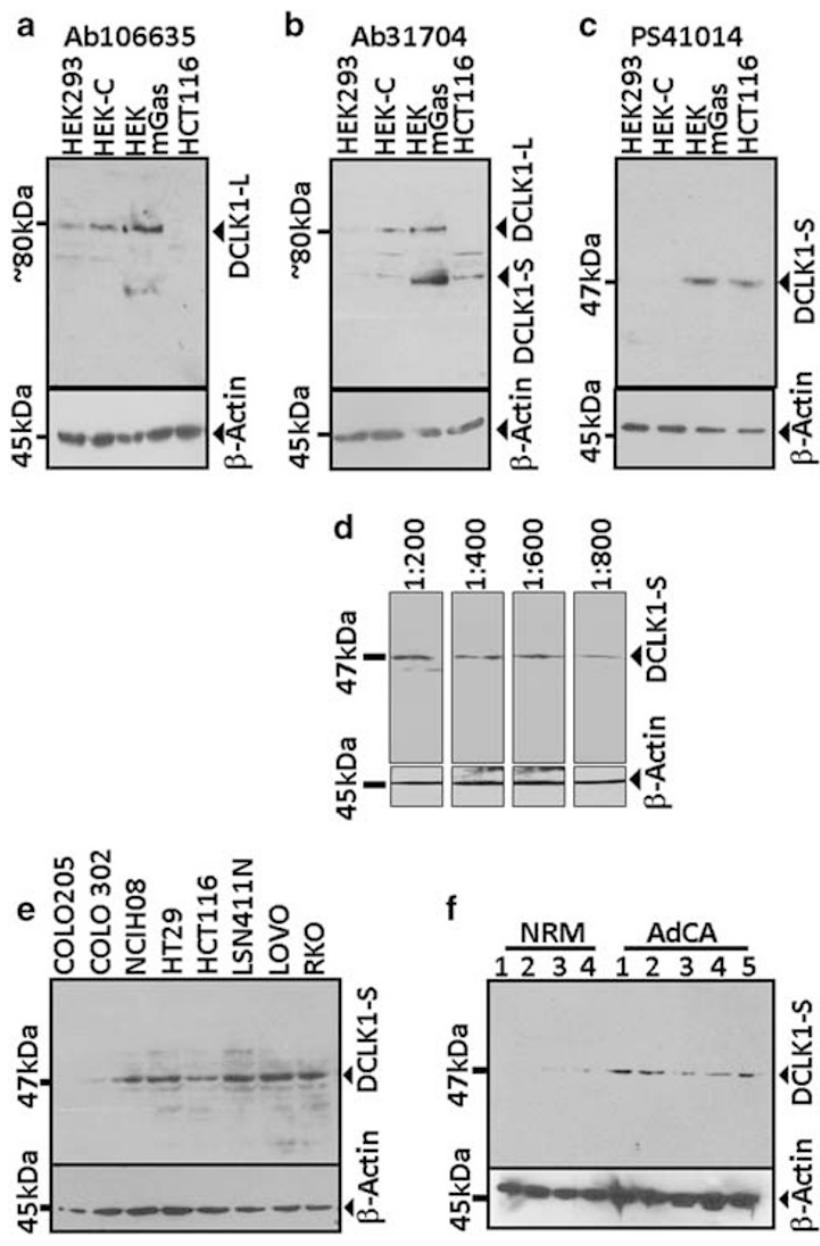

Figure 1 Specificity of PS41014-Ab for detecting DCLK1-S. Cellular lysates were prepared from several cell lines or tissue samples and processed for western blot (WB) analysis with antibodies, which either detects only the L-isoform of DCLK1 (a), using the commercially available Ab (Ab106635), or detects both the L/S-isoforms of DCLK1, using Ab31704 (b), or using the S-specific Ab (PS41014), generated by our laboratory (c-f). The cellular lysates processed for WB analysis in $(\mathbf{a}-\mathbf{c})$ were from the indicated cell lines. Representative WB data from cellular lysates of HCT116 cells, used at the indicated dilutions, are shown in (d). Representative WB data from the indicated human colon cancer cell lines are shown in (e). Representative data from either normal colonic mucosa (NRM) or adenocarcinoma (AdCA) patient samples are presented in (f); the corresponding patients from whom the NRM and AdCA samples were obtained are listed in Supplementary Table 1A. The PS41014-Ab used in (c-f) detected a single band of $47 \mathrm{kDa}$ protein, unlike the commercially available Abs, which appeared to also detect degraded fragments of the $\mathrm{L}$-isoform ( $\mathbf{a}$ and $\mathbf{b}$ ). Relative levels of $\beta$-actin were measured in the same samples, as a loading control, and are shown in (a-f).

L/S-isoforms, as shown in Figure 1b. The common antibody confirmed the presence of L-isoform in the two normal cell lines, S-isoform in HCT116 cells, and both S/L-isoforms in HEKmGAS cells, as expected (Figure 1b). The common antibody, like the long specific $\mathrm{Ab}$, detected other bands of proteins in these cell lines, which either represent degraded fragments of L-isoform or cross-reactivity with other unknown proteins. The short specific Ab generated by us, 
detected only one band of S-isoform with the expected molecular mass $(47 \mathrm{kDa})$, and did not detect any protein bands in normal epithelial cell lines (HEK293, HEKC; Figure 1c), validating the specificity of PS41014-Ab. For results presented in Figures $1 \mathrm{a}-\mathrm{c}$, equivalent concentration of the three antibodies was used $(\sim 5 \mathrm{ng} / \mathrm{ml})$ for detecting DCLK1 isoforms in $50 \mu \mathrm{g}$ of the total cellular lysates. Thus, PS41014-Ab represents the only antibody generated, which specifically detects S-isoform of DCLK1 (isoform 2), without cross-reacting with L-isoforms of DCLK1, to the best of our knowledge.

We next characterized sensitivity of PS41014-Ab for detecting DCLK1-S protein in cellular lysates of HCT116 cells (Figure 1d). The PS41014-Ab detected DCLK1-S in HCT116 cells at different dilutions ranging from 1:200 $(12.5 \mathrm{ng} / \mathrm{ml})$ to $1: 800(3.1 \mathrm{ng} / \mathrm{ml}$; Figure $1 \mathrm{~d})$. At concentrations lower than 1:800, the detection of DCLK1-S was significantly reduced (data not shown). Based on the results presented in Figure 1d, an optimal dilution of 1:500 (5 ng/ml) of PS41014$\mathrm{Ab}$ was chosen for $\mathrm{WB}$ analysis of lysate samples.

\section{Use of PS41014-Ab for Detecting DCLK1-S Isoform in Human Colon Cancer Cell Lines (hCCCs) and Patient Samples by Western Blot Analysis}

We have previously reported that $>80 \%$ of hCCCs express DCLK1-S isoform, and none of the hCRC cell lines examined by us expressed the L-isoform, since the $5^{\prime}$-promoter of $\mathrm{h} D C L K 1$ gene is hypermethylated and epigenetically silenced in all the colon cancer cell lines. ${ }^{12}$ In the current studies, we used PS41014-Ab to confirm the presence/absence of DCLK1-S in CRC cell lines, previously reported to be either positive or negative for the S-transcript. ${ }^{12}$ Representative data from eight cell lines are presented in Figure 1e. Seven cell lines which express S-transcript ${ }^{12}$ were positive for the S-protein band; COLO205 cells, negative for DCLK1-S transcript, ${ }^{12}$ were negative for S-protein (Figure 1e). Isogenic clones of COLO205 cells were developed to overexpress either the $S$ or L-isoform, (as described in the 'Materials and Methods' section), and used for further validation of the PS41014-Ab, by western blot analysis of lysates from the clones (Supplementary Figure 2). WB data with the PS41014-Ab, presented in Supplementary Figure 2, confirmed that the $\mathrm{S}$-specific-Ab only detects the S-isoform in the COLO205-SGFP clones, as described above; the Ab did not detect intact or degraded fragments of the L-isoform in COLO205-L-GFP clones, even though the L-isoform was being overexpressed in a colon cancer cell line, providing strong evidence that the PS41014-Ab is specific for the S-isoform. Importantly, the PS41014-Ab did not detect any proteins in the control COLO205-C-GFP clones, confirming the specificity of the antibody generated by us (Supplementary Figure 2).

We next examined whether PS41014-Ab efficiently detects the S-protein in the lysates of patient samples (Figure 1f). We had previously used the common antibody (Ab31704) for detecting DCLK1 isoforms in the same lysates, prepared from normal colonic mucosa and CRC samples of patients, and reported the presence of only the L-isoform $(82 \mathrm{kDa})$ in the selected normal samples, and the presence of only the S-isoform $(47 \mathrm{kDa})$ in the AdCA samples. ${ }^{12}$ In the current studies, PS41014-Ab did not detect any band in the normal colonic mucosal samples (Figure 1f), confirming the absence of S-isoform in normal colons. The AdCA samples from CRC patients, on the other hand, were positive for variable levels of the S-isoform (Figure 1f), as previously reported with the common antibody, ${ }^{12}$ validating the specificity of PS41014-Ab for the S-isoform (isoform 2), with no cross-reactivity with the L-isoform (isoform 1). Having validated the use of PS41014-Ab for specifically detecting S-isoform of DCLK1 by WB analysis, we next examined its usefulness for detecting the S-isoform by immunohistochemical (IHC) analysis.

\section{IHC Results with PS41014-Ab are More Accurate Than IHC Results with Ab31704, for Detecting DCLK1-S Expression in Patient Samples}

Normal colonic mucosal (NRM) samples mainly express the canonical L-isoform of DCLK1, while colonic AdCAs mainly express the S-isoform, with rapid loss/gain of DCLK1-L/S, respectively, in adenomas (Ads). ${ }^{12}$ The samples of NRM, Ads and AdCAs from several patients (Supplementary Table 1) were obtained as described in the 'Materials and Methods' section, and FFPE samples processed for IHC analysis with Ab31704; representative data from NRM/Ad/AdCA samples are presented in Figure 2a. Ab31704 detected DCLK1 (likely DCLK1-L) in a few colonic crypt epithelial cells, but surprisingly also stained some unknown cells in the surrounding matrix of normal crypts (Figure 2a). A significantly higher percentage of cells in Ad samples were positive for DCLK1, but once again Ab31704 detected DCLK1 in surrounding cells in the matrix. Both the intensity and the $\%$ area of cells positive for DCLK1 was increased in AdCA samples, and the presence of DCLK1+ve cells were once again detected in the surrounding matrix, using the 31704-Ab (Figure 2a). Five separate areas of each section/slide $\times 2$ slides/ sample, was analyzed by ImageJ, and converted to a red scale. The red scale intensity represents the actual staining of the samples after normalizing the color intensity, by filtering the background noise. The percent area stained for each sample was calculated as described in the 'Materials and Methods' section, and data from all the samples analyzed is presented as a box plot in Figure 2b. The number of samples $(n)$ is shown. The gender, age, and ethnicity of the patients from whom these samples were obtained are shown in Supplementary Table 1. On an average, the percent area stained with the Ab31704 increased significantly in Ad vs normal samples and in AdCA vs normal and Ad samples.

We next used PS41014-Ab for staining the patient samples (Figure 3). Representative IHC data from one sample each is presented in Figure 3a. Unlike the results with Ab31704, neither the epithelial component nor the mesenchymal (matrix) component of the normal colonic mucosal samples 
a

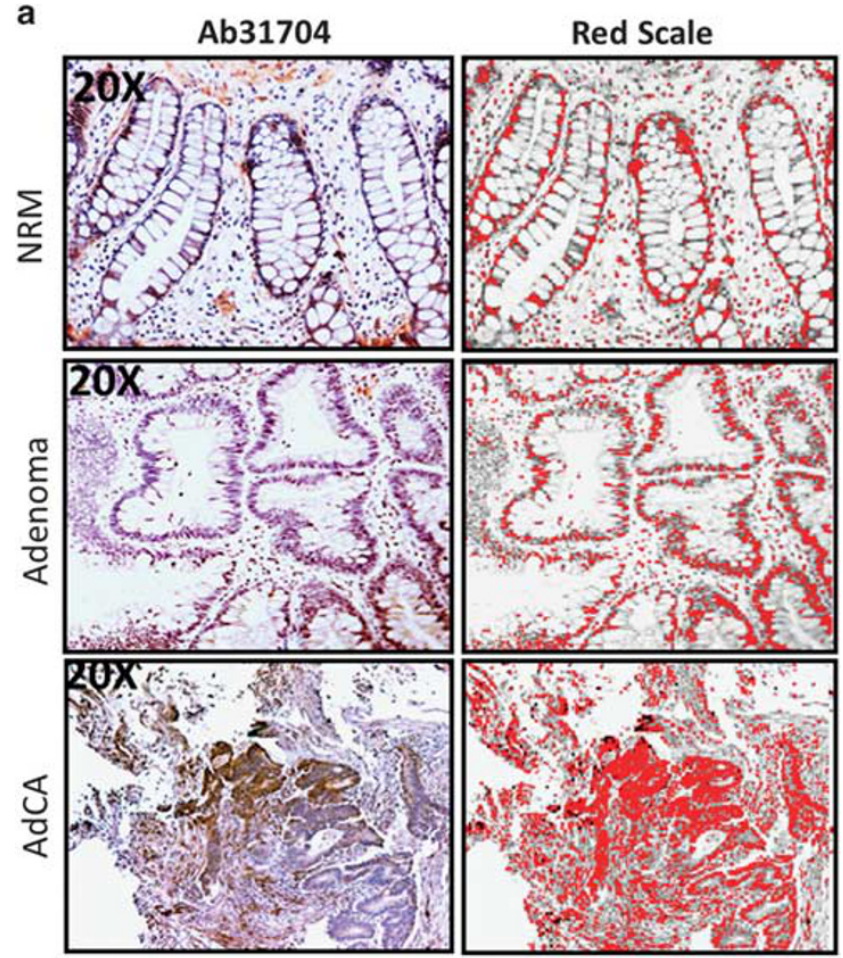

b

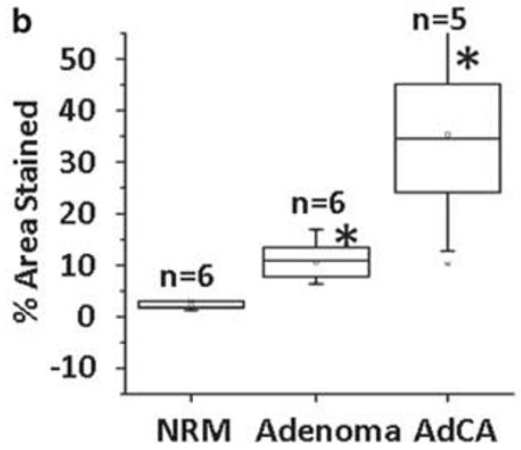

a
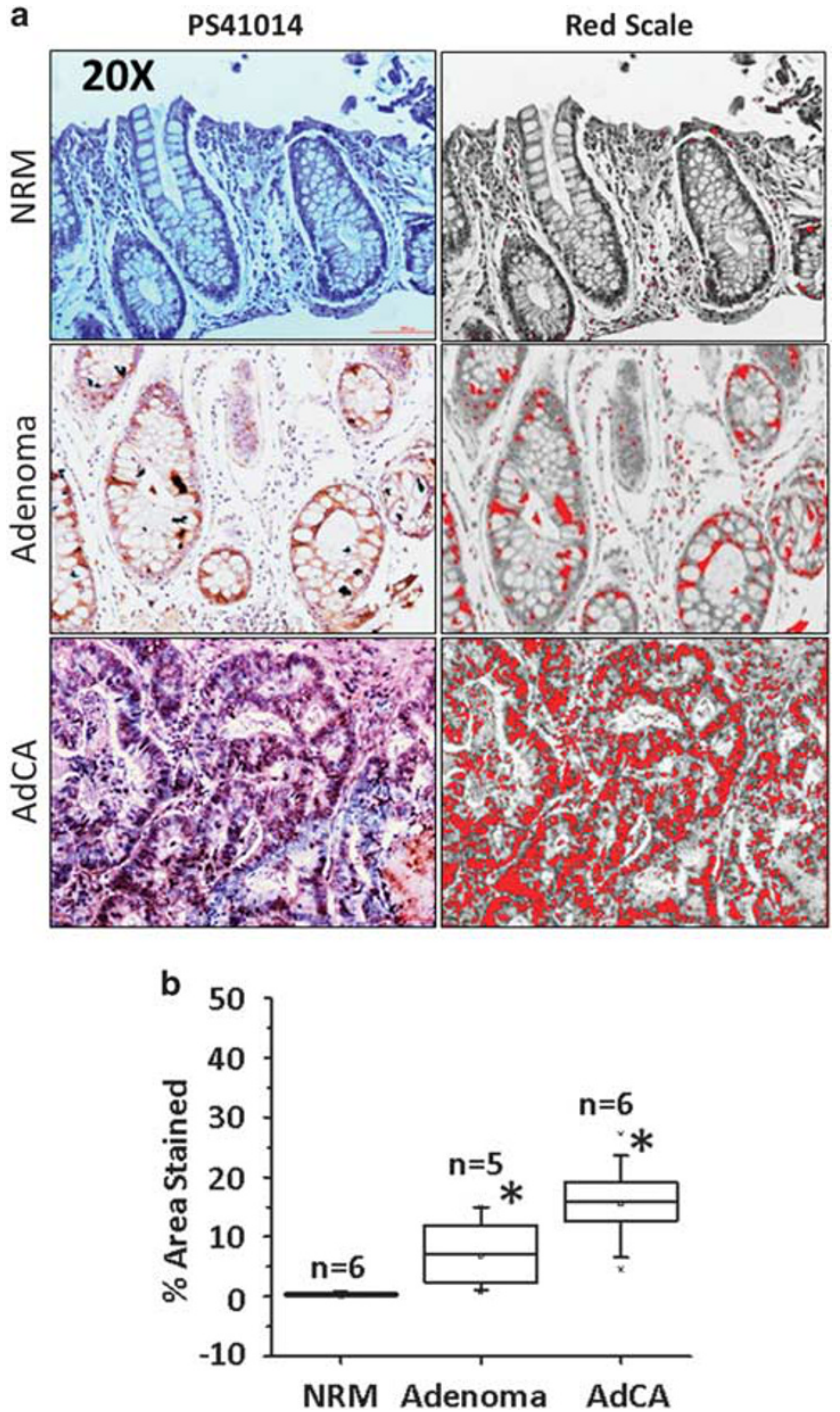

Figure 2 Immunostaining of patient samples with Ab31704. (a, b) Formalin-fixed paraffin-embedded (FFPE) samples from either the normal colonic mucosa (NRM), adenomatous polyps (Ads), or adenocarcinomas (AdCAs) were obtained from the colons of patients, as described in the 'Materials and Methods' section, and the patient-associated information is provided in Supplementary Table 1B. Staining of representative sections of NRM, Ad, and AdCA is presented in (a). The staining data were analyzed by ImageJ, and specific staining converted to the red scale (right-hand side panels) for quantifying the data, as described in the 'Materials and Methods' section. The \% areas stained of the sections from all the patient samples analyzed are presented as box plots in (b). The number of patient samples $(n)$ analyzed are shown for each group. ${ }^{*} P<0.05$ vs normal values.

stained for DCLK1 with the PS41014-Ab, confirming specificity of the antibody, and the absence of S-isoform in all cell types present within the normal colonic mucosa (Figure 3a). The AdCA samples were variably positive for the short isoform (Figure 3a). The \% area stained was calculated for all the samples, as described above, and is presented as box plots in Figure 3b. Unlike the data with Ab31704, none of the

normal samples examined demonstrated staining for DCLK1$\mathrm{S}$ with the PS41014-Ab, strongly validating the specificity of the antibody generated by our laboratory. Importantly, we learned from the current studies that DCLK1-S is not expressed by non-epithelial cells, both in the normal colonic mucosa and in the tumors (Ads/AdCAs; Figure 3a). However, data with 31704-Ab strongly suggests that DCLK1-L is likely expressed by non-epithelial cells, such as neuronal cells, known to express DCLK1-L, ${ }^{36,37}$ and perhaps other, as yet, 
unknown cell types. On an average, DCLK1-S staining with PS41014-Ab, significantly increased in Ads/AdCAs vs normal samples, in the order of AdCAs > Ads > normal mucosa. On an average, the staining of AdCAs with PS41014-Ab, was lower than that with Ab31704 (Figure 2b vs Figure 3b), suggesting the possibility that Ab31704 may be detecting increased expression of the L-isoform in non-epithelial component of AdCA samples; the latter is an intriguing possibility which needs to be further examined. It is, however, important to state that while the NRM samples examined with the common Ab31704 and S-specific PS41014-Ab (Figures 2 and 3) were identical, the Ad/AdCA samples were from different patients, owing to limited availability of sample size. Thus based on our findings so far, results with PS41014-

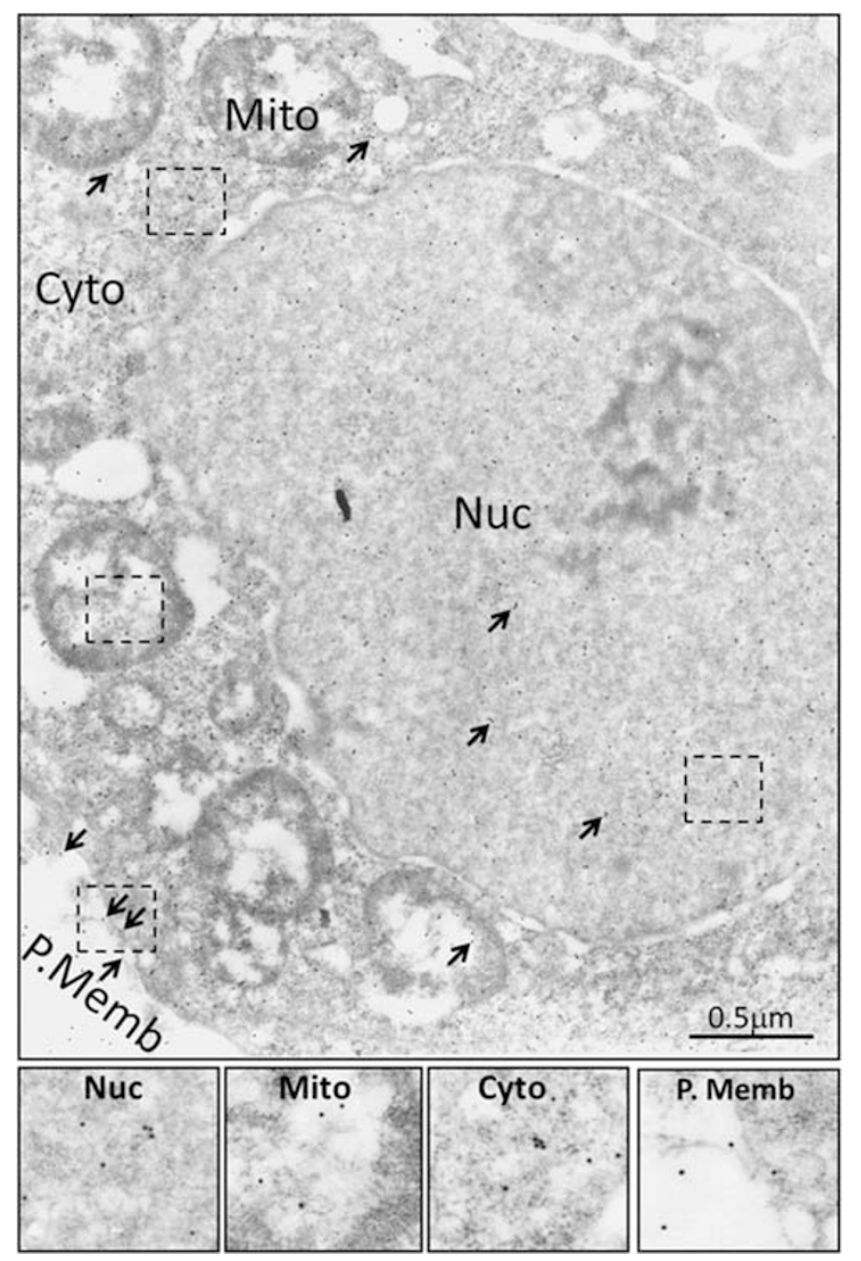

Figure 4 Immuno-electron microscopic (IEM) analysis of staining for DCLK1-S in subcellular fractions of HCT116 cells, using PS41014-Ab. HCT116 cells were processed for IEM analysis as described in the 'Materials and Methods' section, using PS41014-Ab. Gold-labeled second antibody was used for detecting PS41014-Ab, bound to DCLK1-S in the cells. Arrows demonstrate the presence of DCLK1-S staining in subcellular fractions of nucleus (Nuc), mitochondria (Mito), cytosol (Cyto), and plasma membranes (P.Memb). The boxed insets, shown within the dotted outline in the different subcellular fractions were computer enhanced, and are presented at the bottom panels for clarity.
$\mathrm{Ab}$ are expected to provide more accurate information regarding relative expression levels of the S-isoform (specifically expressed by CSCs), at different stages of coloncarcinogenesis, while results with the common antibody (Ab31704) will include detection of L-isoform in normal epithelial cells and other, as yet, unknown cells in the matrix of colonic mucosa. Accurate assessment of relative expression of DCLK1-S by Ad/AdCA samples is important, as we have previously reported that patients expressing high levels of DCLK1-S transcript had an overall worse prognosis than patients expressing relatively low levels of the S-transcript. ${ }^{12}$ On the other hand, relative expression levels of L-transcript (isoform 1) were not associated with overall survival of the patients (unpublished data from our laboratory). As our studies strongly suggest that the L-isoform is not expressed by CSCs, but is expressed by normal epithelial cells and other unknown cell types, it is not surprising that relative levels of L-isoform did not correlate with survival of CRC patients.

Even though the short isoform is $>98 \%$ homologous with the C-terminal end of the long isoform, ${ }^{12}$ the S-isoform lacks the double-cortin domains, which is required for binding microtubules. ${ }^{36,37}$ In preliminary studies, we recently reported that colon cancer cells overexpressing the S-isoform were significantly more invasive than isogenic cells overexpressing the L-isoform. ${ }^{38}$ We therefore hypothesized that the subcellular localization of the S-isoform may be different from that of the L-isoform, and examined localization of the two isoforms by electron microscopy (EM) of isogenic colon cancer cells, which overexpressed GFP-tagged L- (COLO205L-GFP) or S-isoform (COLO205-S-GFP); control clones expressed GFP from the control vector (COLO205-C). COLO205 colon cancer cell line was chosen for these experiments, as it is one of the few hCCCs which does not express either $\mathrm{L}$ or $\mathrm{S}$-isoform, ${ }^{12}$ and can be cloned to express either isoform, as described above. Both the specificity of PS41014-Ab and differences in subcellular localization of the two isoforms was examined by EM, as described below.

\section{Specificity of PS41014-Ab for Detecting DCLK1-S in Colon Cancer Cells by Immuno-Electron Microscopy}

To further confirm the specificity and usefulness of PS41014$\mathrm{Ab}$ for detecting the presence of DCLK1-S by IEM, PS41014$\mathrm{Ab}$ was first used for immuno-electron microscopy (IEM) staining of an S-expressing cell line (HCT116; Figure 4). Surprisingly, we discovered that DCLK1-S is not only present on plasma membranes (P.Memb) and within cytosol (Cyto) of HCT116 cells, but is also present in the nucleus (Nuc) and mitochondrial (Mito) fractions of the cells (Figure 4). To visualize the staining better, the indicated insets in the various subcellular fractions of the cells have been computer enhanced and shown at the bottom of Figure 4. The COLO205-C-GFP cells (which do not express either S/Lisoform of DCLK1), ${ }^{12}$ on the other hand, were negative for DCLK1-S staining with the PS41014-Ab (Supplementary Figure 2). Similarly the HEK293 cells which only express 
the L-isoform were negative for staining with PS41014-Ab, using the IEM method (data not shown). We next examined possible differential subcellular localization of L- vs S-isoforms using IEM, as described below.

\section{The DCLK1-S Isoform Localizes to the Nucleus and Mitochondrial Fractions of the Cells at Significantly Higher Levels Than the DCLK1-L Isoform}

To accurately define differences in the subcellular localization of L- vs S-isoforms, rather than using PS41014-Ab (which can only detect the S-isoform), we used anti-GFP-Ab, for IEM analysis. The anti-GFP-Ab was first used to confirm expression of the L- $v s$ S-isoforms of DCLK1 in the isogenic clones of COLO205 cells (Figure 5a). COLO205-L-GFP clones expressed the expected molecular mass $(\sim 110 \mathrm{kDa})$ of DCLK1-L protein fused to GFP. The COLO205-S-GFP clones expressed the expected molecular mass $(\sim 84 \mathrm{kDa})$ of $\mathrm{S}$ protein fused to GFP. The control clones (COLO205-C-GFP) expressed GFP alone with the expected molecular mass of $\sim 36 \mathrm{kDa}$ (Figure $5 \mathrm{a}$ ). GFP-Ab was next used for IEM analysis of the three isogenic clones, and the data are presented in Figures 6-8. At least 10 separate cells from each clone were analyzed for quantifying the relative localization of L- vs S-isoform in subcellular compartments of nucleus, mitochondria, cytosol, and plasma membranes, and the data from all the sections are presented as bar graphs in Figure 5b. Based on the results presented in Figures 5-8, it can be stated with some confidence, that the S-isoform likely accumulates in the mitochondrial and nuclear compartments at significantly higher concentrations (levels) than the L-isoform, whereas the localization of the S-isoform appears to be significantly reduced on the plasma membranes of the cells, compared with that of the L-isoform. Similarly, in neuronal cells, cleavage of DCLK1-L protein by calpain, was reported to result in the preferential localization of the shorter cleaved protein (lacking the double-cortin domains) to the nucleus of the cells, unlike the intact protein, ${ }^{31}$ resembling our findings with the S-isoform. Localization of the DCLK1 proteins to the mitochondria, however, has not been reported previously. Many of these differences may reflect the differences in the concentration of microtubules in these various subcellular fractions, as the L-isoform binds microtubules..$^{36,37}$ As expected, the overexpressed GFP appears to mainly localize within the cytosol fraction of the COLO205-C-GFP clones (Figures $5 \mathrm{~b}$ and 6 ), providing further validation for the observed presence of $\mathrm{S} / \mathrm{L}$-isoforms in several organelles within the cells, unlike the control GFP protein.

The COLO205-S-GFP clones were also processed for WB analysis (as discussed above), and IEM analysis using the PS41014-Ab. Data from IEM studies with PS41014-Ab are presented in Figure 9, and once again demonstrate that PS41014-Ab detected the presence of S-isoform in all four subcellular fractions. The bar graph in Figure $5 \mathrm{c}$ compares the detection of S-isoform with either the GFP-Ab (Figure 8) or PS41014-Ab (Figure 9). As can be seen, the subcellular distribution of the S-isoform was similar in the subcellular fractions of COLO205-S-GFP clones, using either antibody (Figure 5c), further validating the specificity of PS41014-Ab for IEM analysis as well. For reasons unknown, the PS41014$\mathrm{Ab}$ does not detect S-isoform by immunofluorescence (IF), and we are in the process of optimizing the conditions for using this antibody for other applications. The PS41014-Ab, however, was very useful for immunoprecipitation of the S-isoform, along with its many binding partners, as confirmed by proteomic analysis (unpublished data from our laboratory). The optimal concentrations of the PS41014$\mathrm{Ab}$ required for detecting the S-isoform in different assays is presented in Supplementary Table 2.

As the PS41014-Ab was specific for the S-isoform, and specifically marked CSCs, we hypothesized that higher levels of S-isoform (CSCs) at the early stages of polyp growths may allow identification of high-risk patients, who may be at risk of developing CRCs within shorter time periods. As proof of principle, we conducted a pilot retrospective study, and assigned patients to either low- or high-risk arms of the study, as described in the 'Materials and Methods' section, and examined possible differences in the expression of S-isoform in the polyps of high- $v s$ low-risk patients, as described below.

\section{The Benign/Premalignant Growths (Hps/Ads) at Initial and Follow-Up Colonoscopies, from the High-Risk Patients Express Significantly Higher Levels of DCLK1-S Compared with Polyps from Low-Risk Patients}

As a pilot study, polyps from initial and follow-up colonoscopies were obtained from high- $v s$ low-risk patients as described in the 'Materials and Methods' section. The age, gender, and ethnicity of all the patients, whose samples were obtained from the Department of Pathology as FFPE blocks, are presented in Supplementary Tables 3A and B. The type of polyps and size (whenever available), at the time of initial and subsequent follow-up colonoscopies, are also presented in Supplementary Tables $3 \mathrm{~A}$ and B. The FFPE blocks were processed for making slides with two/three sections per slide by routine histological methods, as published previously, ${ }^{32,33}$ and stained with PS41014-Ab as described in the 'Materials and Methods' section. Representative IHC data from polyps of representative patients at initial and follow-up colonoscopies from the low- and high-risk groups are presented in Figures 10a and $\mathrm{b}$, respectively. The staining data from all the patients were quantified by either, (i) imageJ and red scale method, described above, and presented as \% area stained in Figures $10 \mathrm{c}$, or (ii) by counting \% epithelial cells stained, wherein at least 300 cells were counted per sample at 40X, and data presented as \% cells stained in Figure 10d. The total number $(n)$ of polyps analyzed in the high- and low-risk patients (as shown in Supplementary Tables 3A and B) per colonoscopy are shown above each bar graph (Figures 10c and d). At each colonoscopy, the \% area/cells stained per polyp were significantly higher in the high-risk $v s$ the low-risk patients $(P<0.05 v s$ data presented in the corresponding 

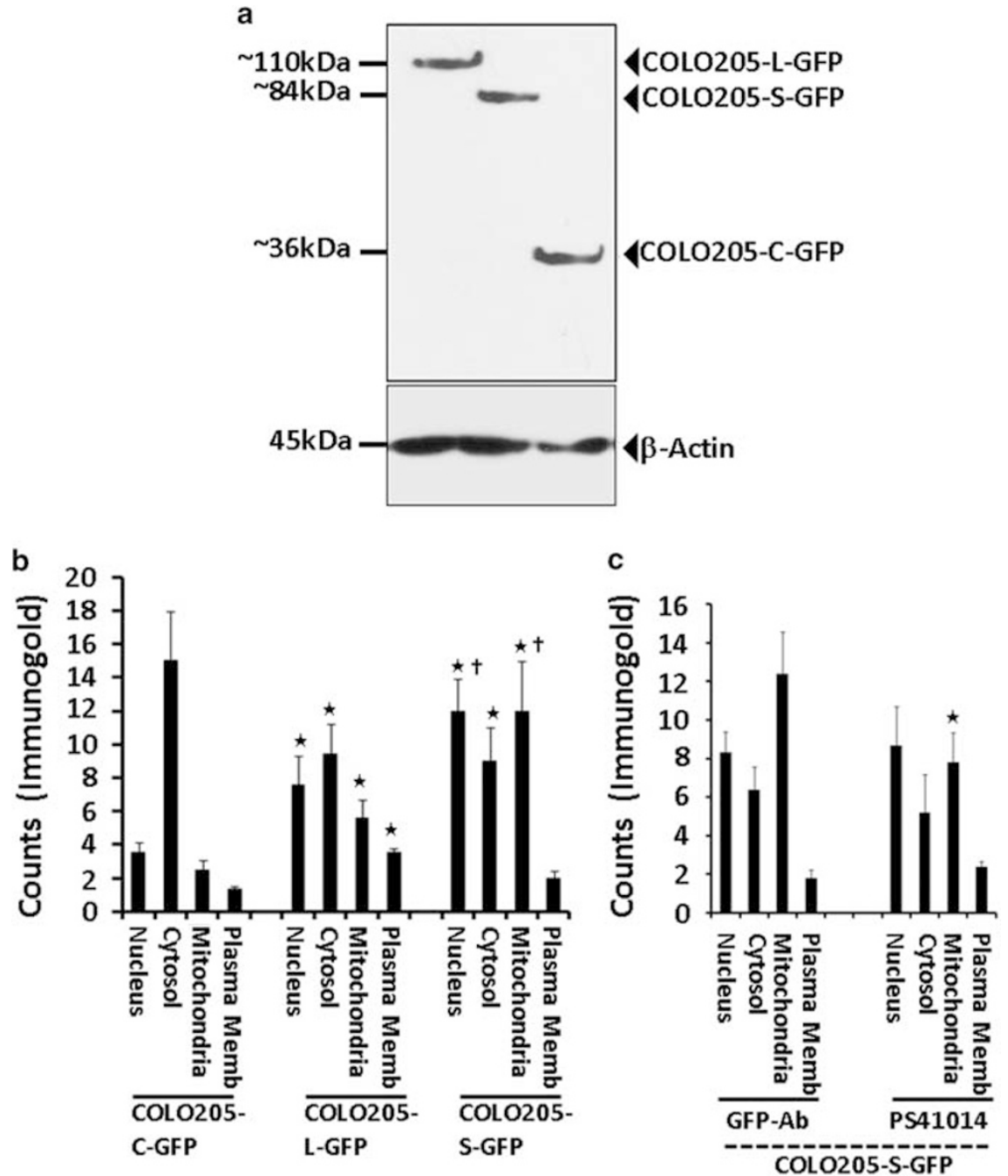

Figure 5 (a) Western blot confirmation of COLO205 clones, expressing GFP-tagged DCLK1-L/S-isoforms, with the expected molecular mass of 110 and $84 \mathrm{kDa}$, respectively, as shown. The control clones (COLO205-C-GFP) demonstrated only the expression of GFP ( 36 kDa). The corresponding $\beta$-actin levels in the cellular lysates of the indicated clones is shown at the bottom of the WB panel. GFP antibody was used for WB analysis of the clones. (b) Subcellular distribution of L- vs S-isoforms of DCLK1, as analyzed by IEM. The colon cancer cell line, COLO205, which does not express either the L- or S-isoform of DCLK1, was sub-cloned to express either the L- or S-isoforms of DCLK1, fused with GFP, and processed for IEM analysis using anti-GFP-Ab, and detected with the gold-labeled second antibody, as described in the 'Materials and Methods' section; control clones expressing only GFP (COLO205C-GFP) were similarly processed. Several sections of the IEM processed clones were analyzed for calculating the relative distribution of the immunegold-labeled proteins in the four subcellular fractions of nucleus, cytosol, mitochondria, and plasma membranes (P.Memb). The data from at least 10-20 separate cells, from three to four separate sections are presented as Mean \pm s.e.m. of counts/cell from each subcellular fraction from each clone. ${ }^{*} P<0.05$ vs corresponding control values from COLO205-C-GFP clones. ${ }^{\dagger} P<0.05$ vs the corresponding values from the COLO205-L-GFP clones. As can be seen, the S-isoform was more heavily located in the nucleus and mitochondria of the cells compared with the L-isoform while lower levels of DCLK1-S vs $-\mathrm{L}$ were observed to be associated with plasma membranes of the cells. (c) Comparison of the subcellular localization of DCLK1-S by IEM analysis using either GFP-Ab or PS41014-Ab. The COLO205-S-GFP clones were processed for EM analysis, and the subcellular localization of DCLK1-S was detected by using either GFP or PS41014-Ab as indicated. The relative localization of DCLK1-S in the subcellular fractions was counted as described above, and the data from the total number of cells analyzed are presented as mean \pm s.e.m. in each bar graph. Other than the mitochondrial fraction, the subcellular localization of DCLK1-S was similar, whether detected with GFP-Ab or PS41014-Ab. The slightly higher detection of DCLK1-S in the mitochondrial fractions with GFP-Ab, compared with PS41014-Ab may reflect the fact that GFP was fused at the N-terminal end of DCLK1-S, which may have masked the binding of PS41014-Ab to the unique amino-acid epitope of the S-isoform at the N-terminal end.

green bars in Figures 10c and d). At least two or more polyps were analyzed per colonoscopy from each patient for the data presented in Figures 10c and d. The data from all the polyps obtained at each colonoscopy, from a representative patient in the high- and low-risk groups, are also presented in
Figure 10e, to demonstrate the stark difference in the staining pattern of the polyps at each subsequent colonoscopy for high- $v s$ low-risk patients. The pattern of staining suggests that: \% staining of polyps from the low-risk group remains at baseline levels at each follow-up colonoscopy, and may even 


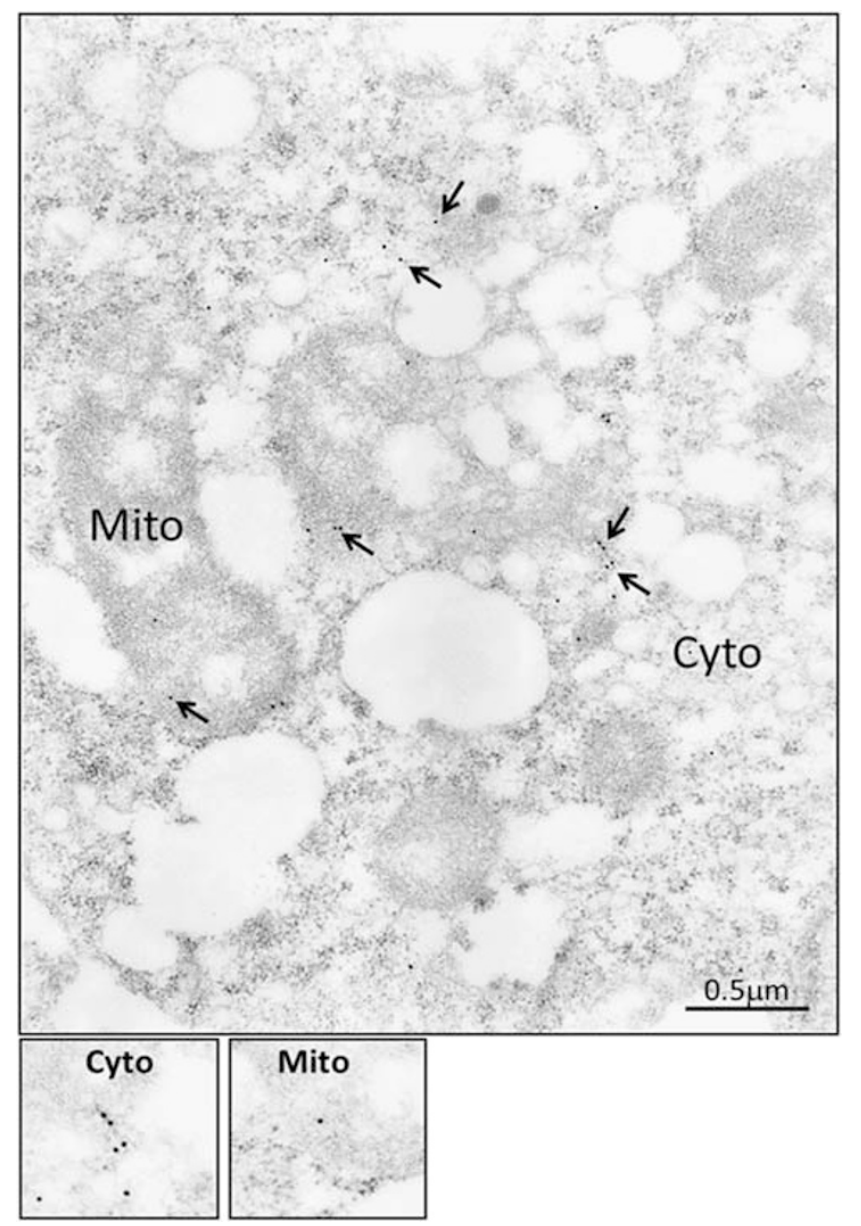

Figure 6 Subcellular localization of control GFP protein in COLO205-CGFP clones by IEM analysis with GFP antibody. The control COLO205 clones were processed for IEM analysis, as described in the legend of Figure 4, but GFP-Ab was used as the primary antibody. The control GFP protein was for the most part present in the cytosolic fraction, as can be appreciated from the quantitative data presented in Figure $5 \mathrm{~b}$.

decrease with advancing age; staining of polyps from the highrisk patients, on the other hand, trends higher at each subsequent colonoscopy (Figure 10e). Importantly the \% staining of the polyps in the high-risk patients was at least two- to threefold higher than that in the polyps from low-risk patients at all time points, and resemble the levels of staining measured in AdCA samples ( 20\%), as shown in Figure 3. The data presented in Figure 3 was obtained from Ads at the time of screening colonoscopies, in a prospective study, with no knowledge of whether these patients developed AdCAs in their lifetime. Thus the \% staining of Ads in Figure 3 likely represents staining from both low- and high-risk patients, overlapping with AdCA-staining data (Figure 3), unlike the data presented in Figure 10, which separates the staining of Ads in the two groups. Thus, based on data presented in Figure 10, on an average, the $\%$ area stained in the polyps of the low-risk patients ranged from $<1 \%$ to $\sim 10 \%$, with an average of $\sim 5 \%$. The $\%$ staining of the polyps from high-risk

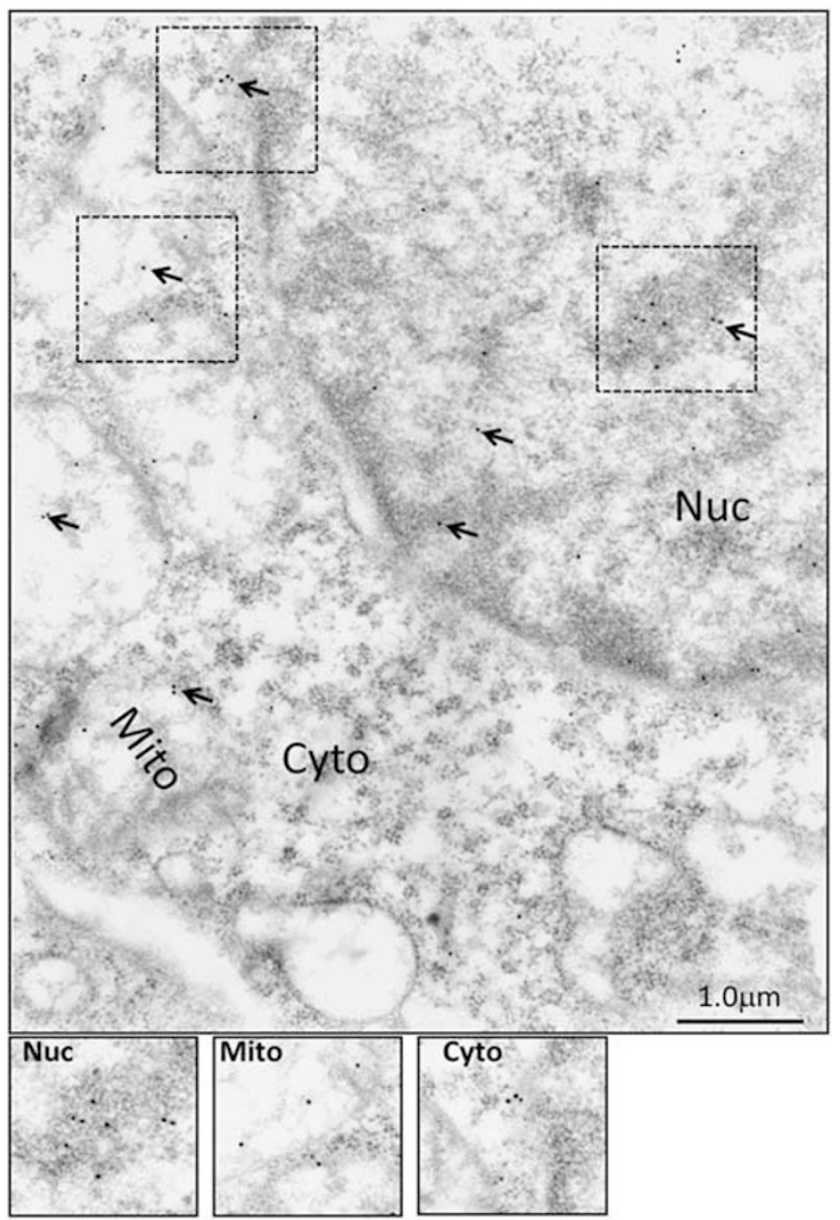

Figure 7 Subcellular distribution of the long isoform of DCLK1 in COLO205-L-GFP clones, processed with GFP antibody. The COLO205-LGFP clones were processed for IEM analysis as described in the 'Materials and Methods' section, and as outlined in the legend of Figure 6, using GFP-Ab. The boxed insets from different subcellular fractions of the cell are enhanced at the bottom of the figure. The relative distribution of DCLK1-L isoform in the different subcellular fractions was quantified as described in the legend of Figure 5, and is presented in Figure $5 \mathrm{~b}$.

patients, on the other hand, ranged from $\sim 10 \%$ to $30-40 \%$ with an average of $\sim 20 \%$. These findings suggest, for the first time, that the PS41014-Ab can be used at the time of index/ screening colonoscopy for assigning low or high risk to the patients, irrespective of the size/number and morphology of the polyps, as currently used for recommending follow-up colonoscopies. For example, even though patient \#246 (Supplementary Table 3A) from the low-risk group had frequent colonoscopies, the polyps remained poorly positive for DCLK1-S, all through the follow-up colonoscopies (Figure 10e). On the other hand, patient \#254 (Supplementary Table 3B) from the high-risk group developed AdCAs within 10 years, even though at the time of initial colonoscopy in 2006, the patient was not classified as a highrisk patient based on number/morphology and pathology of the polyps, discovered at that time. Thus, the additional use of 


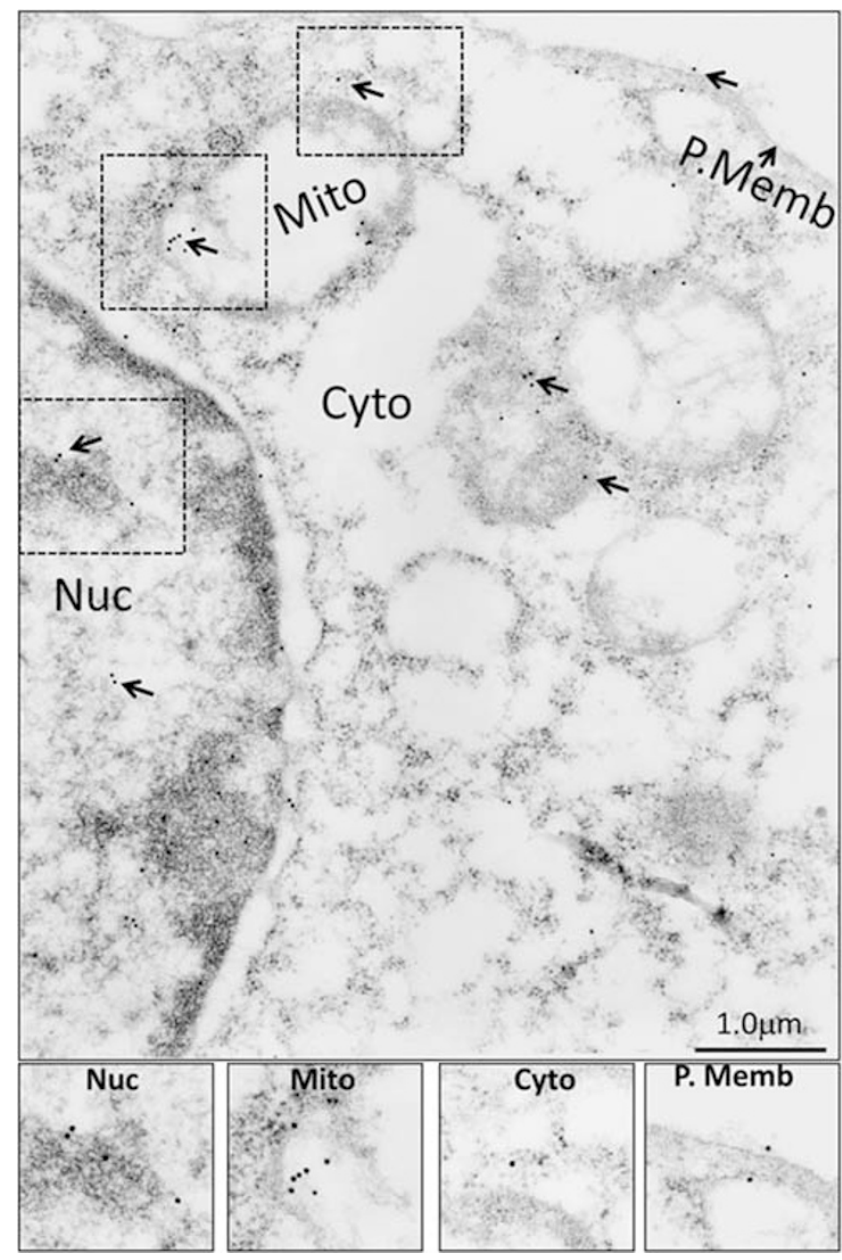

Figure 8 Subcellular localization of DCLK1-S isoform in COLO205-S-GFP clones, stained with GFP antibody. The COLO205-S-GFP clones were processed for IEM analysis with GFP-Ab as described in the legend of Figure 6 , and the insets demonstrating the presence of DCLK1-S in the different subcellular fractions is enhanced and presented at the bottom of the figure. The relative distribution of DCLK1-S isoform was quantified as described in the legend of Figure $5 b$, and is presented as bar graphs in Figures $5 b$ and $c$.

the PS41014-Ab for IHC staining of the resected polyps at the time of screening colonoscopy will help guide the physicians for more accurate follow-up recommendations, and serve as an important prognostic biomarker at an early stage of the disease, at a time when there are no other available serum or fecal markers that can help identify the presence of CSCs and help assess the risk of the patients for developing CRCs. The results in Figure 10 additionally suggest that the resection of polyps in the low-risk group, especially during initial colonoscopies, appears to provide significant protection to the patients from developing advanced growths in their lifetime, while resection of the polyps during colonoscopies in patients at high risk, does not, by itself, provide protection to the patients from developing AdCAs. It may, therefore, be important to not only resect all polyps at early stages in both the low- and high-risk groups, but also to identify high-risk

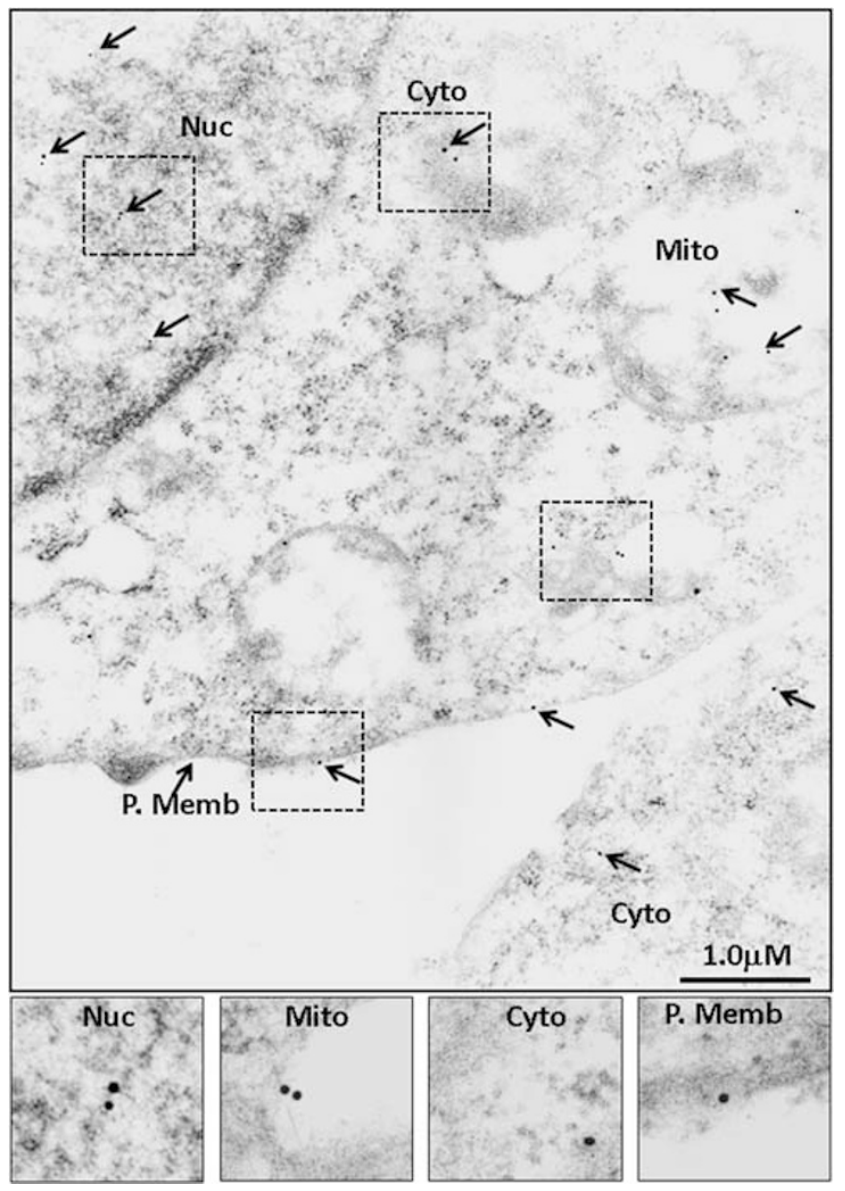

Figure 9 Subcellular distribution of DCLK1-S in COLO205-S-GFP clones by IEM analysis with PS41014-Ab. The COLO205-S-GFP clones were processed for IEM analysis with PS41014-Ab as well, to compare the relative distribution of staining with either the GFP or PS41014-Ab. The insets from the different subcellular fractions are amplified and presented at the bottom of the figure, and the relative distribution of DCLK1-S, using either one of the two antibodies is presented in Figure $5 \mathrm{c}$.

patients at an early stage and perhaps use additional preventative strategies for protecting the high-risk patients from developing advanced growths within shorter intervals. Identification of CSC populations using the PS41014-Ab may allow the identification of high-risk patients at a very early stage, and give an opportunity to develop preventive and interventional strategies for these patients.

\section{DISCUSSION}

Based on the results of the current study, we report generation of a unique monospecific polyclonal antibody (PS41014) against the short (S) isoform (isoform 2) of DCLK1, which specifically marks cancer stem cells (CSCs) in adenomas (Ads) and adenocarcinomas (AdCAs) of human colons. The specificity of the antibody $(\mathrm{Ab})$ for detecting only the short isoform, with no cross-reactivity to the canonical long (L) isoform of DCLK1, was demonstrated by using isogenic clones of either human embryonic epithelial cells (HEKC, HEKmGAS) or human colon cancer cells (hCCCs; 
a

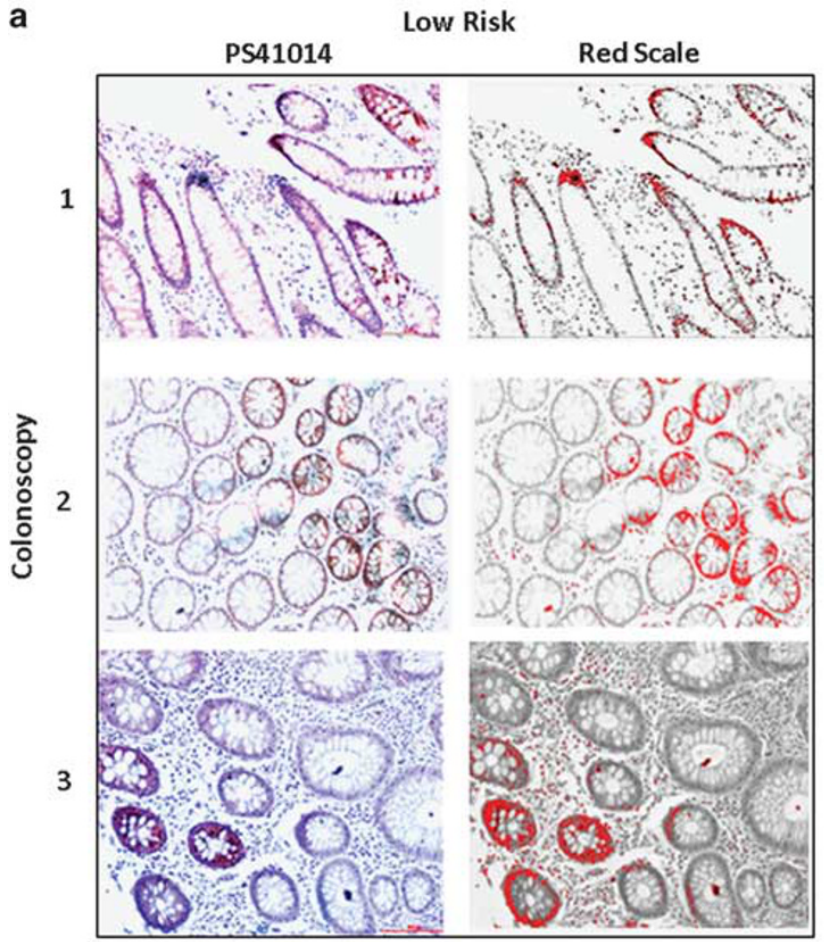

b

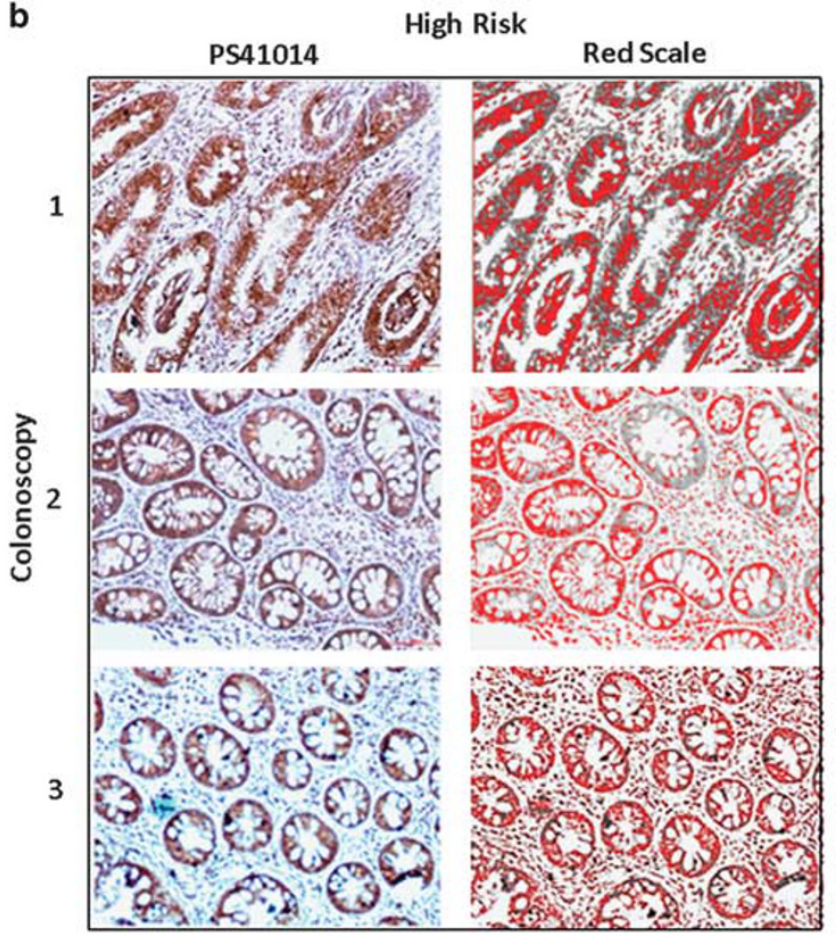

c

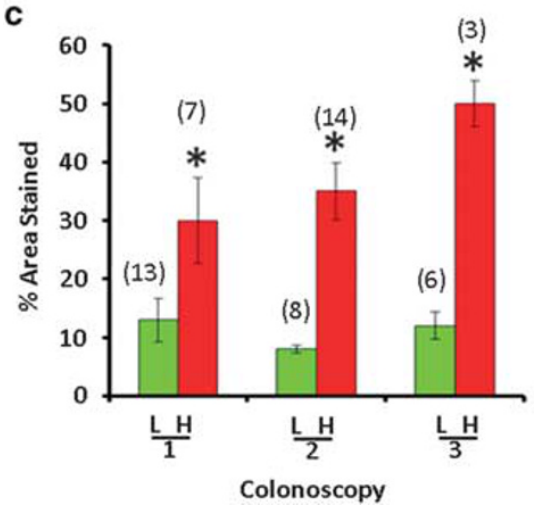

d

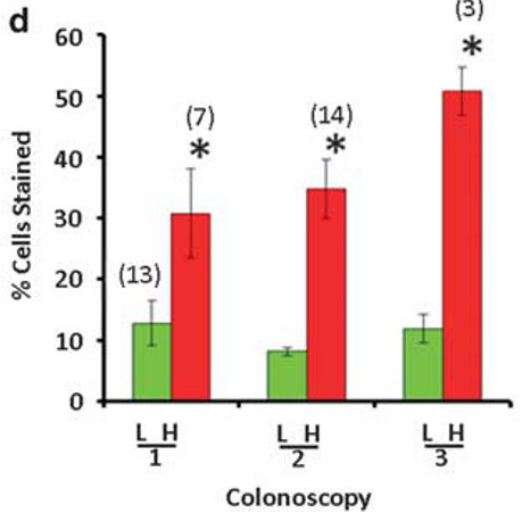

e

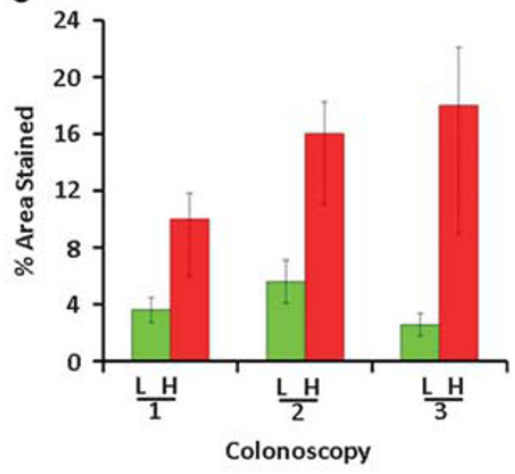

Figure 10 Relative levels of DCLK1-S staining in the adenomas from low- vs high-risk patients in a pilot retrospective study. Patients who had multiple colonoscopies at UTMB were separated in the two groups of low vs high risk, based on the absence or formation of AdCAs in the colon, within 10 years of initial colonoscopy, as described in the 'Materials and Methods' section. (a, b) Available FFPE samples of adenomas, from the identified patients (Supplementary Tables 3A and B), from the initial colonoscopy and follow-up colonoscopies were obtained as described in the 'Materials and Methods' section and processed for IHC staining with PS41014-Ab. IHC staining of representative polyps from a patient, that were removed at initial colonoscopy (colonoscopy \#1) and subsequent follow-up colonoscopies (colonoscopies \#2 and 3) are presented in (a) (representative low-risk patient) and (b) (representative high-risk patient). The red scale conversion of the specific staining data is shown in the right-hand panels. (c-e) The staining data from the total number $(n)$ of polyps from the indicated patients in Supplementary Table 3 are presented as either \% area stained (bar graphs in c) or \% epithelial cells stained (bar graphs in $\mathbf{d}$ ), wherein the data from the low-risk patients is compared with the data from high-risk patients at colonoscopy $\# 1-3$. The total number of polyps analyzed is presented at each time point at the top of each bar graph (c and $\mathbf{d}$ ). The data in each bar graph are the mean \pm s.e.m. of the indicated number $(n)$ of samples. ${ }^{*} P<0.05$ between corresponding data for low-risk patients (green bars) in (c and $\mathbf{d}$ ). The data for all the polyps from a single representative patient, at each colonoscopy for a low-risk and a high-risk patient, are also presented as bar graphs in (e).

COLO205-S, COLO205-L), which either expressed the L or S-isoform of DCLK1 (Figures 1 and 5). The specificity of PS41014-Ab for detecting DCLK1-S was further confirmed by screening several hCCCs. We have previously reported that none of the hCCCs screened by us expressed DCLK1-L (isoform 1), whereas 80\% expressed DCLK1-S, and 10-20\% (including COLO205) did not express either L/S-isoforms. ${ }^{12}$ In the current studies, COLO205 cells were confirmed to be negative for the presence of DCLK1-S when examined with the PS41014-Ab (Figure 1e). The specificity of PS41014-Ab for the S-isoform was further validated by WB of normal human colonic mucosal (NRM) samples and AdCA samples 
from CRC patients. NRM samples were previously analyzed by qRT-PCR and reported to express only DCLK1-L; ${ }^{12}$ same samples were used in the current studies, and confirmed to be negative for the S-isoform by WB analysis with PS41014-Ab (Figure 1f). The AdCA samples, on the other hand, previously reported to express only DCLK1-S by qRT-PCR analysis, ${ }^{12}$ demonstrated a single band of $\sim 47 \mathrm{kDa}$, representing DCLK1-S in western blots, when probed with PS41014-Ab (Figure 1f). Hammond et al, ${ }^{39}$ have similarly reported the presence of only an $\sim 47 \mathrm{kDa}$ DCLK1 protein in western blots of hCCCs, when probed with the commercially available $\mathrm{Ab}$, which detects both L/S-isoforms of DCLK1. Thus, the generation of the DCLK1-S-specific Ab, as reported in the current studies, will allow investigators for the first time to specifically measure the expression of DCLK1-S protein, without resorting to electrophoretic separation of the isoforms when using the common Ab.

As both the epithelial and the extraepithelial components of NRM do not express DCLK1-S isoform, ${ }^{12}$ the staining of the NRM samples with PS41014-Ab was almost nil (Figure 3a), further validating the use of PS41014-Ab for IHC detection of DCLK1-S. However, the commercially available common $\mathrm{Ab}$ against DCLK1 (which detects both L/S-isoforms), stained several cells in both the epithelial and non-epithelial components of normal mucosa (Figure 2a), suggesting that DCLK1-L is expressed by other cell types, besides epithelial cells, in the normal colonic mucosa. The PS41014-Ab stained $<1 \%$ area of normal samples (Figure 3), whereas both the common $\mathrm{Ab}$ (Ab31704; Figure 2) and the commercially available $\mathrm{Ab}$ against the long isoform (Ab106635; data not shown), stained $\sim 3-6 \%$ of the normal samples.

The specificity of the PS41014-Ab was also validated for staining of the S-isoform at the EM level, by analyzing cells devoid of DCLK1-S expression (HEK293), or by analyzing isogenic clones of COLO205 cells, engineered to overexpress either the L- or the S-isoform of DCLK1 (Figures 5-9). Besides establishing the specificity of the PS41014-Ab, using many different platforms for detecting DCLK1-S, with no cross-reactivity to the L-isoform, the sensitivity of the PS41014-Ab was also examined (Figure 1c; Supplementary Table 2). The sensitivity of the PS41014-Ab for optimally detecting DCLK1-S in the different assay platforms, was established in terms of $\mathrm{ng} / \mathrm{ml}$ (Supplementary Table 2). We were thus able to obtain significant titers of an S-specific $\mathrm{Ab}$ in rabbits, using only the six unique amino-acid epitope at the $\mathrm{N}$-terminal end of the S-isoform, even though the rest of the $\mathrm{S}$-isoform was homologous with the C-terminal end of the L-isoform.

An important finding of the current study was that while the S-isoform is mainly expressed by the epithelial component of Ads and AdCAs (Figure 3), the L-isoform appeared to be expressed by several cell types, including normal colonic crypt cells and other unknown cell types located in the matrix of the normal colonic mucosa/colonic tumors. Neuronal cells in the brain are known to express DCLK1-L. ${ }^{36,37}$ It thus appears likely that DCLK1-L is also expressed by enteric neurons. The specific cell types, which express DCLK1-L in the nonepithelial component of either normal human colonic mucosa or colonic tumors, however, needs to be further examined.

As expected, the relative expression levels of DCLK1-S, on an average, were significantly higher in Ads of the colon, compared with that in the normal colonic mucosa (Figure 3). Similarly, relative expression levels of the S-isoform, on an average, were higher in AdCAs, compared with that in Ads and normal colonic mucosa (Figure 3), suggesting that on an average, \% cells expressing DCLK1-S increased in colon tumors during colon-carcinogenesis, compared with that in the normal mucosa (Figure 3). Increased expression of DCLK1 in both primary tumors and distant metastatic tumors, compared to that in the normal colonic mucosa, has been reported, ${ }^{28,29}$ using the commercially available common $\mathrm{Ab}$, which, however, does not distinguish between L/S-isoforms of DCLK1. As the common antibody detects both isoforms in the epithelial and extraepithelial components of the tumors, the relative staining of Ads/AdCAs measured with the common $\mathrm{Ab}$, on an average, appeared to be higher compared with that measured with the PS41014-Ab (Figures 2 and 3), suggesting that besides the S-isoform, the L-isoform expression may also increase in the tumors, especially at the AdCA stage, the significance of which needs to be examined.

A novel finding of the current studies was that $\mathrm{L} / \mathrm{S}$-isoforms of DCLK1 were not only present in the cytosol and plasma membranes of colon cancer cells, but a significant fraction of the DCLK1 protein was found in the nuclear and mitochondrial fractions, as determined by EM analysis (Figures 4-9). HEK293 cells, which do not express the S-isoform of DCLK1, did not stain with PS41014-Ab at the EM level (data not shown). But the PS41014-Ab stained DCLK1-S in HCT116 cells (Figure 4), validating the use of PS41014-Ab for specific detection of DCLK1-S by EM analysis, as well. An interesting finding was that the levels of S-isoform in the nuclear and mitochondrial fractions was much higher than that of L-isoform, in cancer cells, with very low presence at the plasma membranes, unlike the L-isoform (Figures 4-9). The subcellular localization of DCLK1-S was similar in HCT116 and COLO205-S-GFP cells (Figures 4 and 8), suggesting that both endogenously (HCT116) and exogenously (COLO201S-GFP) expressed S-isoform traffics similarly and localizes within the same cellular organelles at equivalent percentages. Importantly, the PS41014-Ab demonstrated a very similar pattern of subcellular staining as the GFP-Ab in the COLO205-S-GFP clones (Figures 5c, 8 and 9), once again validating the specificity of the PS41014-Ab for the short isoform.

The molecular size of L/S-isoforms of DCLK1 are significantly different, as the S-isoform lacks the two double-cortin (DCX) domains present at the $\mathrm{N}$-terminal 
end of the L-isoform. The absence of DCX domains in DCLK1-S may preclude the S-isoform from binding the microtubules, as described for L-isoform. ${ }^{36,37}$ The crystal structure of L-isoform was recently published, ${ }^{40}$ but the crystal structure of the S-isoform remains unknown. Absence of the microtubule-binding domain in the S-isoform may allow differential localization of the two proteins, as suggested by the EM results (Figure 5b). Differences in localization of the $\mathrm{S} / \mathrm{L}$-isoforms may lend itself to differences in the biological activity of the two isoforms as recently reported by us in a preliminary study. ${ }^{38}$ Colon cancer cells overexpressing the S-isoform (COLO205-S-GFP) were found to be significantly more invasive, in vitro and in vivo, compared with the isogenic cells overexpressing the L-isoform (COLO205-L-GFP). ${ }^{38}$

Hyperplastic/adenomatous (Hp/Ad) growths are the most common neoplastic sporadic growths found during colorectal screening of patients, with no genetic predisposition. ${ }^{3}$ The time to CRCs from Ads is predicted to vary from 10 to 22 years. ${ }^{4}$ At age $60,30-40 \%$ of patients are positive for colonic Ads, but the lifetime cumulative incidence of CRC is 5.5\%, suggesting that most Ads will not progress to cancer. ${ }^{2}$

With aging populations in Europe, United States, Japan, China, the number of CRC cases are expected to significantly increase in future years. ${ }^{9}$ In addition, a significant increase in the incidence of CRCs has been noted in young adults. ${ }^{41}$ It is extrapolated that by 2030 , incidence of CRCs will rise by 90 $124 \%$ for patients $20-34$ years and by $27-46 \%$ for patients aged 35-49 years, based on a retrospective study from 1975 through $2010 .^{41}$ However, a small but significant decline in the incidence of CRCs in patients aged 50+, has been noted due to increased screening/surveillance of older patients. ${ }^{41}$ 'Field cancerization' is characterized by genetic/epigenetic alterations in normal-appearing colonic tissues in patients with Ads/CRCs, leading to increased risk of synchronous/ metachronous primary tumors (reviewed in ref. 42). Genetic mutations are rare in normal/hyperplastic cells; however, epigenetic changes occur at early stages. Changes in the landscape of DNA methylations, resulting from gradual dysregulation of the genome (epigenetic drift) is associated with aging, causing 'field effects' in CRCs (reviewed in ref. 42). Epigenetic changes are being captured by DNA/ noncodingRNA(miRNA) tests of fecal/blood samples from patients, for identifying patients with CRCs, including the FDA-approved Cologuard test (reviewed in ref. 42). However, these tests do not identify patients with neoplastic growths (Hps/Ads) and have limited sensitivity for identifying premalignant growths or sessile-serrated polyps (reviewed in ref. 42). The miRNA-based biomarker panels, developed so far, are inconsistent (reviewed in ref. 42) and none of the panels are approved for guiding follow-up colonoscopies, which remain the gold standard used for detecting CRCs or advanced growths in patients.

Size/numbers/dysplasia of polyps remains the only reliable predictors of risk, and for guiding time to follow-up colonoscopies; but these features are not precise and majority of the patients subjected to follow-up colonoscopies will never develop cancer. ${ }^{5-7}$ To assess whether specific neoplastic growths predispose patients to malignant growths, predictive gene signatures have been developed, ${ }^{42-44}$ which require frozen biopsies of variable quality. Persistent technical difficulties are noted with multiplexed qRT-PCR platforms. ${ }^{45}$ Identification of patients, positive for high-risk Ads, at initial/screening colonoscopies, using blood/stool based biomarkers has thus far remained elusive (reviewed in ref. 42). A significant drawback of DNA/RNA/miRNA-based biomarkers is that the sample examined may not be representative of the complete polyp, and quality of collection has remained a significant issue. IHC analysis, on the other hand is cost effective, can potentially cover the entire field of the fixed specimen and can be analyzed by the pathologist while assessing morphological/pathological criteria, much like the analysis of estrogen receptor/HER2neu oncogene, used for prognostic purposes in breast cancer patients. A study has validated the use of colonoscopy with morphological/ pathological analysis of polyps, as a cost effective method of prevention and identification of high-risk patients, than all other fecal/blood tests developed so far. ${ }^{46}$ Very few biomarkers, other than progastrin, have been reported for identifying patients at risk for developing advanced growths using IHC assay of the $\mathrm{Ad} / \mathrm{Hp}$ samples. ${ }^{47} \mathrm{We}$ hypothesized that antibody-based assay(s), which identify transformed stem cells, specific for colon cancers, such as DCLK1-S, will allow a pathologist in any setting, to significantly improve the predictive value of morphological/pathological criteria.

To test our hypothesis, we used the specific PS41014-Ab for IHC analysis of Ads, discovered at the time of index/screening colonoscopies, in a pilot retrospective study, in order to determine whether staining with this antibody could accurately identify high-risk patients (who eventually developed AdCAs within 10 years of initial colonoscopy). Based on the results of our pilot retrospective study, the PS41014-Ab was found to stain Ads from initial and follow-up colonoscopies of high-risk patients, at significantly higher levels than Ads from low-risk patients (Figure 10), suggesting that PS41014-Ab could be used as an additional tool for more accurately identifying high-risk patients. Identification of CSC populations, using the PS41014-Ab may allow identifying the high-risk patients at a very early stage, and give an opportunity to develop preventive and interventional strategies for these patients. In addition, we must also keep in mind that polypectomies performed during colonoscopies are curative in most patients-utilizing the tissues that are already obtained during these procedures to provide additional prognostic information for patients will be of great benefit.

Colonoscopy is the most commonly performed medical procedure in the United States with 14 million procedures in 2003 alone. ${ }^{46}$ Of the millions who get repeat colonoscopies as recommended, only $1-2 \%$ actually develop CRCs. ${ }^{46}$ 
Therefore, molecular biomarkers are critically required for identifying the high-risk patients at early time points, so that high-risk patients not only receive more frequent colonoscopies, but are also subjected to additional preventive/interventional strategies, based on the findings of our pilot retrospective studies. Stem cells are critically required for maintaining both normal/abnormal growths, malignant transformation of stem cells is a critical step towards malignancy; increased CSC populations can lead to invasion and seeding of metastatic growths at shorter intervals. ${ }^{15,16,38}$ So far there are no biomarkers which specify CSCs in colonic growths. Molecular markers developed so far, for analyzing serum/fecal samples from patients, do not identify high-risk patients, at an early stage, when patients are positive for only polyps. ${ }^{42-44}$ In addition, there are no tests/biomarkers available for identifying high-risk patients, at the time of index/screening colonoscopy, to determine which patients may be at risk of developing A-Ads/CRCs within 10-15 years. Based on the results of our pilot retrospective study, identification of high-risk patients at an early time point, based on the pattern of staining for DCLK1-S, at initial and follow-up colonoscopies, can be used to trigger additional preventative/interventional strategies, besides frequent followup colonoscopies.

In conclusion, we have generated a DCLK1-S-specific polyclonal $\mathrm{Ab}$ using the six amino-acid epitope, unique to the S-isoform, and have validated the specificity of the PS41014$\mathrm{Ab}$ by WB/IHC/IEM. We have previously reported that analysis of the relative expression levels of the S-isoform by qRT-PCR analysis can serve as an independent diagnostic and prognostic marker for identifying CRC patients at risk of poor overall survival. ${ }^{12}$ In the current studies, we demonstrate that the S-specific $\mathrm{Ab}$ can probably identify patients at the early time points of index/screening colonoscopy, who may be at high risk for developing CRCs. The use of PS41014-Ab is significantly more accurate than using commercially available Abs, which measures both L/S-isoforms, given that DCLK1-L is not expressed by human cancer stem cells, but is expressed by many different cell types in the extraepithelial component of the tumors, as well as by the normal colonic epithelial cells.

Supplementary Information accompanies the paper on the Laboratory Investigation website (http://www.laboratoryinvestigation.org)

\section{ACKNOWLEDGMENTS}

This work was supported by the National Institutes of Health (http://www.nih. gov/) grants CA97959 and CA97959S1 to PS. The funders had no role in the study design, data collection and analysis, decision to publish, or the preparation of the manuscript.

\section{AUTHOR CONTRIBUTIONS}

SS generated all the data with the help of VLP's laboratory and MO and BSL; $\mathrm{RAO}, \mathrm{BSL}, \mathrm{HLS}$, and GL helped to identify the patient samples from two hospital databases and retrieve the FFPE blocks from the pathology department; HLS helped to confirm the pathology of all the samples; MO and BSL helped SS to analyze all the IHC data, while VLP helped SS to analyze the IEM data; HLS and VLP helped PS to interpret the data and write the manuscript, with the help of all the authors; PS directed all the studies. The authors acknowledge the technical help received from Julie W Wen and Zhixia Ding, in Dr Popov's laboratory, for the IEM studies.

\section{DISCLOSURE/CONFLICT OF INTEREST}

The authors declare no conflict of interest.

1. Siegel R, Naishadham D, Jemal A. Cancer statistics, 2013. CA Cancer J Clin 2013;63:11-30.

2. Atkin WS, Saunders BP, British Society for Gastroenterology, et al. Surveillance guidelines after removal of colorectal adenomatous polyps. Gut 2002;51(Suppl 5):V6-V9.

3. Bujanda L, Cosme A, Gil I et al. Malignant colorectal polyps. World J Gastroenterol 2010;16:3103-3111.

4. Force USPST. Screening for colorectal cancer: U.S. Preventive Services Task Force recommendation statement. Ann Intern Med 2008;149: 627-637.

5. Winawer SJ, Zauber AG, Fletcher RH, et al. Guidelines for colonoscopy surveillance after polypectomy: a consensus update by the US MultiSociety Task Force on Colorectal Cancer and the American Cancer Society. CA Cancer J Clin 2006;56:143-159; quiz 84-5.

6. van Stolk RU, Beck GJ, Baron JA, et al. Adenoma characteristics at first colonoscopy as predictors of adenoma recurrence and characteristics at follow-up. The Polyp Prevention Study Group. Gastroenterology 1998;115:13-18.

7. Huang CS, O'brien MJ, Yang S, et al. Hyperplastic polyps, serrated adenomas, and the serrated polyp neoplasia pathway. Am J Gastroenterol 2004;99:2242-2255.

8. Atkin WS, Morson BC, Cuzick J. Long-term risk of colorectal cancer after excision of rectosigmoid adenomas. N Engl J Med 1992;326:658-662.

9. Amonkar MM, Hunt TL, Zhou Z, et al. Surveillance patterns and polyp recurrence following diagnosis and excision of colorectal polyps in a medicare population. Cancer Epidemiol Biomarkers Prev 2005;14: 417-421.

10. Imperiale TF, Glowinski EA, Lin-Cooper C, et al. Five-year risk of colorectal neoplasia after negative screening colonoscopy. N Engl J Med 2008;359:1218-1224.

11. Sarkar S, Swiercz R, Kantara C, et al. Annexin A2 mediates up-regulation of NF-KB, $\beta$-catenin, and stem cell in response to progastrin in mice and HEK-293 cells. Gastroenterology 2011;140:583-595.

12. O'Connell MR, Sarkar S, Luthra GK, et al. Epigenetic changes and alternate promoter usage by human colon cancers for expressing DCLK1-isoforms: Clinical Implications. Sci Rep 2015;5:14983.

13. Nakanishi $Y$, Seno H, Fukuoka A, et al. Dclk1 distinguishes between tumor and normal stem cells in the intestine. Nat Genet 2013;45: 98-103.

14. Westphalen CB, Asfaha S, Hayakawa Y, et al. Long-lived intestinal tuft cells serve as colon cancer-initiating cells. J Clin Invest 2014;124: 1283-1295.

15. Kantara C, O'Connell M, Sarkar S, et al. Curcumin promotes autophagic survival of a subset of colon cancer stem cells, which are ablated by DCLK1-siRNA. Cancer Res 2014;74:2487-2498.

16. Sarkar S, Kantara C, Ortiz I, et al. Progastrin overexpression imparts tumorigenic/metastatic potential to embryonic epithelial cells: phenotypic differences between transformed and nontransformed stem cells. Int J Cancer 2012;131:E1088-E1099.

17. Vedeld HM, Skotheim Rl, Lothe RA, et al. The recently suggested intestinal cancer stem cell marker DCLK1 is an epigenetic biomarker for colorectal cancer. Epigenetics 2014;9:346-350.

18. Vedeld HM, Andresen K, Eilertsen IA, et al. The novel colorectal cancer biomarkers CDO1, ZSCAN18 and ZNF331 are frequently methylated across gastrointestinal cancers. Int J Cancer 2015;136:844-853.

19. Powrózek T, Krawczyk P, Nicoś M, et al. Methylation of the DCLK1 promoter region in circulating free DNA and its prognostic value in lung cancer patients. Clin Transl Oncol 2016;18:398-404.

20. Singh P, O'Connell M, Sarkar S. Epigenetic regulation of human DCLK-1 gene during colon-carcinogenesis: clinical and mechanistic implications. Stem Cell Investig 2016;28:51.

21. Bailey JM, Alsina J, Rasheed ZA, et al. DCLK1 marks a morphologically distinct subpopulation of cells with stem cell properties in preinvasive pancreatic cancer. Gastroenterology 2014;146:245-256. 
22. Westphalen $C B$, Takemoto $Y$, Tanaka $T$ et al. Dclk1 defines quiescent pancreatic progenitors that promote injury-induced regeneration and tumorigenesis. Cell Stem Cell 2016;18:441-455. Comments from Singh et al, regarding inaccuracies in this publication, are published and available at this link: http://www.cell.com/cell-stem-cell/comments/S1934-5909 (16)30003-0.

23. Chandrakesan $\mathrm{P}, \mathrm{May} \mathrm{R}, \mathrm{Qu} \mathrm{D}$, et al. Dclk1+ small intestinal epithelial tuft cells display the hallmarks of quiescence and self-renewal. Oncotarget 2015;6:30876-30886.

24. May $R$, Riehl TE, Hunt $C$, et al. Identification of a novel putative gastrointestinal stem cell and adenoma stem cell marker, doublecortin and CaM kinase-like-1, following radiation injury and in adenomatous polyposis coli/multiple intestinal neoplasia mice. Stem Cells 2008;26: 630-637.

25. May R, Sureban SM, Hoang N, et al. Doublecortin and CaM kinase-like-1 and leucine-rich-repeat-containing G-protein-coupled receptor mark quiescent and cycling intestinal stem cells, respectively. Stem Cells 2009;27:2571-2579.

26. Sureban $S M$, May $R$, Ramalingam $S$, et al. Selective blockade of DCAMKL-1 results in tumor growth arrest by a Let-7a MicroRNAdependent mechanism. Gastroenterology 2009:137:649-659.

27. Kantara C, O'Connell MR, Luthra G, et al. Methods for detecting circulating cancer stem cells (CCSCs) as a novel approach for diagnosis of colon cancer relapse/metastasis. J. Lab Invest 2015;95:100-112.

28. Gagliardi G, Goswami M, Passera R, et al. DCLK1 immunoreactivity in colorectal neoplasia. Clin Exp Gastroenterol 2012;5:35-42.

29. Gagliardi G, Moroz K, Bellows CF. Immunolocalization of DCAMKL-1, a putative intestinal stem cell marker, in normal colonic tissue. Pathol Res Pract 2012;208:475-479.

30. Sarkar S, O'Connell MR, Singh P. Differential dose effects of curcumin/ analogs in long (L) versus short $(\mathrm{S})$ isoforms of a cancer stem cells marker, DCLK1 in colon and pancreatic cancer cells: biological impact. Proceedings of the American Association Cancer Research (AACR) 107th Annual Meeting; 16-20 April 2016; Abstract \#5242, New Orleans, LA, USA.

31. Burgess HA, Reiner O. Cleavage of doublecortin-like kinase by calpain releases an active kinase fragment from a microtubule anchorage domain. J Biol Chem 2001;276:36397-36403.

32. Singh $\mathrm{P}$, Velasco $\mathrm{M}$, Given $\mathrm{R}$, et al. Progastrin expression predisposes mice to colon carcinomas and adenomas in response to a chemical carcinogen. Gastroenterology 2000;119:162-171.

33. Umar S, Sarkar S, Cowey S, et al. Activation of NF-kappaB is required for mediating proliferative and antiapoptotic effects of progastrin on proximal colonic crypts of mice, in vivo. Oncogene 2008;27:5599-5611.
34. Zhang J, Popov VL, Gao S et al. The developmental cycle of Ehrlichia chaffeensis in vertebrate cells. Cell Microbiol 2007;9:610-618.

35. Popov VL, Yu X, Walker DH. The $120 \mathrm{kDa}$ outer membrane protein of Ehrlichia chaffeensis: preferential expression on dense-core cells and gene expression in Ehrlichia coli associated with attachment and entry. Microb Pathog 2000;28:71-80.

36. Lin PT, Gleeson JG, Corbo JC, et al. DCAMKL1 encodes a protein kinase with homology to doublecortin that regulates microtubule polymerization. J Neurosci 2000;20:9152-9161.

37. Shu T, Tseng HC, Sapir T, et al. Doublecortin-like kinase controls neurogenesis by regulating mitotic spindles and $\mathrm{M}$ phase progression. Neuron 2006;49:25-39.

38. O'Connell MR, Sarkar S, Ward D et al. Short (S) isoform of cancer-stemcell marker, DCLK1, is critically required for maintaining proliferative/ tumorigenic potential of human colon cancer cells (hCCCs) independent of DCLK1-L isoform. (Poster of distinction). Proceeding of the Digestive Disease Week, 21-24 May 2016. Gastroenterology 2016;150: S-605.

39. Hammond $\mathrm{DE}$, Mageean $\mathrm{CJ}$, Rusilowicz EV, et al. Differential reprogramming of isogenic colorectal cancer cells by distinct activating KRAS mutations. J Proteome Res 2015;14:1535-1546.

40. Patel O, Dai W, Mentzel $M$, et al. Biochemical and structural insights into doublecortin-like kinase domain 1. Structure 2016;24: 1550-1561.

41. Bailey $C E, H u C Y$, You $Y N$, et al. Increasing disparities in the age-related incidences of colon and rectal cancers in the United States, 1975-2010. JAMA Surg 2015;150:17-22.

42. Okugawa Y, Grady WM, Goel A. Epigenetic alterations in colorectal cancer: emerging biomarkers. Gastroenterology 2015;149:1204-1225.

43. Galamb O, Wichmann B, Sipos F, et al. Dysplasia-carcinoma transition specific transcripts in colonic biopsy samples. PLoS ONE 2012;7: e48547.

44. Drew JE, Farquharson AJ, Mayer CD, et al. Predictive gene signatures: molecular markers distinguishing colon adenomatous polyp and carcinoma. PLoS ONE 2014;9:e113071.

45. Binnicker MJ. Multiplex molecular panels for diagnosis of gastrointestinal infection: performance, result interpretation, and costeffectiveness. J Clin Microbiol 2015;53:3723-3728.

46. Seeff LC, Richards TB, Shapiro JA, et al. How many endoscopies are performed for colorectal cancer screening? Results from CDC's survey of endoscopic capacity. Gastroenterology 2004;127:1670-1677.

47. Do C, Bertrand C, Palasse J, et al. A new biomarker that predicts colonic neoplasia outcome in patients with hyperplastic colonic polyps. Cancer Prev Res (Phila) 2012;5:675-684. 